\title{
Über Dreiecks- und Vieleckssysteme als Träger der Kurve dritter Ordnung.
}

Von Dr. Hermann Oppenheimer, Neustadt a. H. (Rheinpfalz).

\section{Einleitung.}

Denken wir uns in bekannter Weise die Punkte einer $C_{3}$ durch Parameter elliptischer Funktionen dargestellt, derart, da einem Wendepunkte der Parameter 0 zukommt, und seien die Parameter der Eckpunkte irgend eines der $C_{3}$ einbeschriebenen Dreiecks $A B C$ bezw. $a, b, c$; ist nun $K$ irgend ein Funkt der $C_{3}$ mit dem Parameter $k$, so liefern die dritten Schnittpunkte der Geraden $A K, B K, C K$ mit der $C_{3}$ ein weiteres Dreieck $A_{k}^{\prime} B_{k}^{\prime} C_{k}^{\prime}$ mit bezw. den Parametern $-(a+k),-(b+k),-(c+k)$; projizieren wir dieses Dreieck $A_{k}^{\prime} B_{k}^{\prime} C_{k}^{\prime}$ wieder von einem Punkte der $C_{3}$, am einfachsten von dem Wendepunkte mit dem Parameter 0 aus, so erhalten wir das Dreieck $A_{k} B_{k} C_{k}$ mit den Parametern der Eckpunkte bezw. $a+k, b+k, c+k$ (und beliebigen Vielfachen der Periodizitätsmoduln). Lassen wir nun den Projektionspunkt $K$ sich auf der Kurve entlang bewegen, so bekommen wir ein System von Dreiecken $A_{k} B_{k} C_{k}$, die sich mit den Punkten der $C_{3}$ in eine vollständige Analogie bringen lassen; ja mehr noch als das: ich werde zeigen, dab die Mannigfaltigkeit der Punkte $\operatorname{der} C_{3}$ einen speziellen Fall eines Systems ron Dreiecken der geschilderten Art darstellt.

Die Beziehungen und Sätze, zu denen ein solches System von Dreiecken $A_{k} B_{k} C_{k}$ Anlaß gibt, und ihre Vergleichung mit den bekannten Punktsätzen der $C_{3}$ sind der Gegenstand der nachfolgenden Arbeit; den Schluß derselben bildet eine kurze Erörterung der Vieleckssysteme, die eine gleiche Verwertung wie die eben genannten Dreieckssysteme zulassen.

1. Wir wollen ein solches Dreieck $A_{k} B_{k} C_{k}$ mit bezw. den Parametern $a+k, b+k, c+k$ den "Tripelpunkt $k$ " nennen. Verbinden wir nun zwei solche Tripelpunkte $k_{1}$ and $k_{2}$, so geben sie in folgender, eindeutiger Weise Anlaß zu einem weiteren Tripelpunkte $k_{3}$ : Es ist $-\left(a+k_{1}+b+k_{2}\right) \equiv-\left(b+k_{1}+a+k_{2}\right)$ $\left(\bmod \omega, \omega^{\prime}\right)$, wenn $\omega$ und $\omega^{\prime}$ die beiden Moduln der Kurve sind; 
ebenso $-\left(b+k_{1}+c+k_{2}\right) \equiv-\left(b+k_{2}+c+k_{1}\right) ;-\left(c+k_{1}+\right.$ $\left.+a+k_{2}\right) \equiv-\left(c+k_{2}+a+k_{1}\right)$, d. h. $A_{k_{1}} B_{k_{2}}$ und $A_{k_{2}} B_{k_{1}}$ schneiden sich in demselben Punkte $C_{k_{3}}$ der Kurve, $B_{k_{1}} C_{k_{2}}$ und $B_{k_{2}} C_{k_{1}}$ in demselben Punkte $A_{k_{3}}, C_{k_{1}} A_{k_{2}}$ and $C_{k_{2}} A_{k_{1}}$ in demselben Punkte $B_{k_{3}}$. Man erhält also in eindeutiger Weise aus den beiden Dreiecken $A_{k_{1}} B_{\dot{k}_{1}} C_{k_{1}}$ and $A_{k_{2}} B_{k_{2}} C_{k_{2}}$, die dem Dreieckssystem $(a+k$, $b+k, c+k)$ angehören, das Dreieck $A_{k_{3}} B_{k_{3}} C_{k_{3}}$. Dabei ist z. B. das Punktpaar $A_{k_{3}}-B_{k_{3}}$ die Projektion des Punktpaares $A_{k_{2}}-B_{k_{2}}$ von $C_{k_{1}}$ aus oder die Projektion des Punktpaares $A_{k_{1}}-B_{k_{1}}$ von $C_{k_{2}}$ aus ü. s. w.

2. Es ist nun leicht zu erkennen, daß das Dreieck $A_{k_{3}} B_{k_{3}} C_{k_{3}}$ ebenfalls ein Tripelpunkt, $d . h$. in dem System der Dreiecke $(a+k, b+k, c+k)$ enthalten ist. Die Parameter seiner Eckpunkte sind, wie wir gesehen haben, $-\left(b+k_{1}+c+k_{2}\right)$, $-\left(c+k_{1}+a+k_{2}\right),-\left(a+k_{1}+b+k_{2}\right)$ oder, wenn wir $a+b+c$ jedesmal addieren und subtrahieren: $a-\left[a+b+c+k_{1}+k_{2}\right]$, $b-\left[a+b+c+k_{1}+k_{2}\right], c-\left[a+b+c+k_{1}+k_{2}\right]$. Dieser Form der Parameter sieht man sofort an, dah das Dreieck unserem System angehört. Gleichzeitig ergibt sich folgender Satz: Verbinden wir zwei Tripelpunkte $k_{1}$ and $k_{2}$ miteinander, so ergibt sich in eindeutiger. Weise der Tripelpunkt $k_{3}=-(a+b+c+$ $+k_{1}+k_{2}$ ). Die Ähnlichkeit dieses Ergebnisses mit dem Ergebnis der Verbindung einfacher Punkte liegt auf der Hand; die Übereinstimmung in der Form läpt sich, wie wir später sehen werden, dadurch zu einer vollständigen machen, daf wir als Ausgangsdreieck $A B C$ ein solches ausfindig machen, dessen Eckpunktparametersumme $a+b+c=0$ ist. Mit Rücksicht auf diese Ähnlichkeit können wir uns auch so ausdrïcken: Die drei Tripelpunkte $k_{1}, k_{2}, k_{3}$ liegen a u einer Tripelgeraden.

3. Sind die beiden Tripelpunkte $k_{1}$ und $k_{2}$ unendlich benachbart, ist also $k_{1}=k_{2}$, so ist der dritte Tripelpunkt $k_{3}$ der Verbindungslinie von $k_{1}$ und $k_{2}$ der Tangentialpunkt von $k_{1}$; er hat demnach den Parameter $-\left(a+b+c+2 k_{1}\right)$. Betrachten wir nun wieder das Dreieck $A_{k_{1}} B_{k_{1}} C_{k_{1}}$ und das unendlich benachbarte $A_{k_{2}} B_{k_{2}} C_{k_{2}}$, so wird, wie wir in (2) gesehen haben, dieser Tangentialpunkt geliefert durch die Geraden $A_{k_{2}} B_{k_{2}}, B_{k_{1}} C_{k_{2}}, C_{k_{1}} A_{k_{2}}$ oder wegen des Zusammenfallens von $k_{1}$ und $k_{2}$ durch $A_{k_{1}} B_{k_{1}}, B_{k_{1}} C_{k_{1}}$ und $C_{k_{1}} A_{k_{1}}$. Man erhält also zu einem Tripelpunkte $k_{1}$ den Tangentialpunkt $k_{1}^{\prime}$, wenn man die dritten Schnittpunkte der Seiten des Dreiecks $A_{k_{\mathrm{L}}} B_{k_{\mathrm{L}}} C_{k_{\mathrm{k}}}$ bestimmt. Es hat nun auch keine Schwierigkeit, amgekehrt zu einem Tripelpunkte $k^{\prime}$ die vier Tripelpunkte $\mathrm{zu}$ bestimmen, von denen er Tangentialpunkt ist; ist $x$ einer derselben, dann ist also $-(a+b+c+2 x)+p \omega+p^{\prime} \omega^{\prime}=k^{\prime}$ (wobei $p$ und $p^{\prime}$ beliebige ganze Zahlen sind); also ist 


$$
x=-\frac{a+b+c}{2}+\frac{p \omega+p^{\prime} \omega^{\prime}-k^{\prime}}{2}
$$

der Ausdruck bestätigt, daß es vier Punkte $x$ gibt, eine Tatsache, die sich ja auch schon daraus ergibt, daß es bekanntlich vier einer $C_{3}$ einbeschriebene Dreiecke gibt, die einem anderen der $C_{3}$ einbeschriebenen Dreieck umbeschrieben sind. In unserem Falle erhält man die vier Tripelpunkte, wenn man in dem Ausdruck für $x p$ und $p^{\prime}$ bezw. $=0$ und $=1$ setzt. Die vier Parameter sind also:

$$
\begin{gathered}
-\frac{a+b+c}{2}+\frac{k^{\prime}}{2},-\frac{a+b+c}{2}-\frac{k^{\prime}}{2}+\frac{\omega}{2} \\
-\frac{a+b+c}{2}-\frac{k^{\prime}}{2}+\frac{\omega^{\prime}}{2},-\frac{a+b+c}{2}-\frac{k^{\prime}}{2}+\frac{\omega+\omega^{\prime}}{2} .
\end{gathered}
$$

Die Analogie mit der diesbezüglichen Theorie der einfachen Punkte springt wieder in die Augen.

4. Wir ersehen aus den Parametern der vier Tripelpunkte, die denselben Tangentialpunkt haben, auch, dab es drei Systeme konjugierter Tripelpunktpaare gibt. Dem Tripelpunkt $-\frac{a+b+c+k^{\prime}}{2}$ muf man bezw. $\frac{\omega}{2}, \frac{\omega^{\prime}}{2}, \frac{\omega+\omega^{\prime}}{2}$ hinzufügen, um jedesmal einen konjugierten Tripelpunkt zu bekommen. Zu einem beliebigen Tripelpunkte $k_{n}$ gehören also die konjugierten Punkte $k_{n}+\frac{\omega}{2}, k_{n}+\frac{\omega^{\prime}}{2}$, $k_{n}+\frac{\omega+\omega^{\prime}}{2}$. Nehmen wir zwei konjugierte Tripelpunktpaare desselben Systems $k_{n}, k_{n}+\frac{\omega}{2}$ and $k_{m}, k_{m}+\frac{\omega}{2}$, so ist es vielleicht von Interesse, den Parallelismus in dem Verhalten zweier solcher Tripelpunktpaare zu dem zweier gewöhnlicher konjugierter Punktpaare desselben Systems noch einmal durch Konstruktion ersichtlich zu machen. $k_{m}$ und $k_{n}$ liefern als dritten Punkt der Tripelgeraden, zu der sie gehören, wie wir gesehen haben, den Tripelpunkt $-\left(a+b+c+k_{n}+k_{m}\right)$; ebenso ergeben aber auch die Tripelpunkte $k_{n}+\frac{\omega}{2}$ und $k_{n}+\frac{\omega}{2}$, wenn wir sie verbinden, als dritten Tripelpunkt $-\left(a+b+c+k_{n}+k_{s n}+\frac{\omega}{2}+\frac{\omega}{2}\right) \equiv-(a+b+$ $\left.+c+k_{n}+k_{m}\right)\left(\bmod \omega, \omega^{\prime}\right){ }_{2} d$. h. denselben Punkt; anderseits liefern $k_{m}$ and $k_{n}+\frac{\omega}{2}$ den dritten Tripelpunkt $-(a+b+c+$ $\left.+k_{m}+k_{n}+\frac{\omega}{2}\right)$, ebenso wie $k_{n}$ und $k_{i n}+\frac{\omega}{2}$.

Wenn wir von der symbolischen Darstellungsweise absehen, so heißt das also: Die Dreiecke $A_{k_{n}} B_{k_{n}} C_{k_{n}}$ and $A_{k_{m}} B_{k_{m}} C_{k_{m}}$ liefern 
dasselbe dritte Dreieck wie die Dreiecke $A_{k_{n}+\frac{\omega}{2}} B_{k_{n}+\frac{\omega}{2}} C_{k_{n}+\frac{\omega}{2}}$ und $A_{k_{m}+\frac{\omega}{2}} B_{k_{m}+\frac{\omega}{2}} C_{k_{k_{m}}+\frac{\omega}{2}}$, ebenso liefert das Dreieckpaar $A_{k_{n}} B_{k_{n}} C_{k_{n}}$

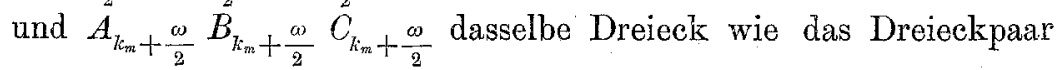
$A_{k_{m}} B_{k_{m_{m}}} C_{k_{m}}$ und $A_{k_{n}+\frac{\omega}{2}} B_{k_{a}+\frac{\omega}{2}} C_{k_{n}+\frac{\omega}{2}}$. Dabei bedeutet das Dreieck $A_{k_{n}} B_{k_{n}} C_{k_{n}}$ den Tripelpunkt $k_{n}$ u. s. w.

Es lassen sich nun alle anderen Eigenschaften konjugierter Punktpaare auf unsere Tripelpunktpaare übertragen. Ist z. B. $k_{m}$ und $k_{m}+\frac{\omega}{2}$ wieder ein solches Paar konjugierter Tripelpunkte, so ist der dritte Schnittpunkt $k_{\text {su }}^{\prime \prime}$ ihrer Verbindungslinie $=-\left(a+b+c+2 k_{\mathrm{m}}\right)+\frac{\omega}{2} ;$ anderseits ist der Tangentialpunkt von $k_{m}$ und $k_{m}+\frac{\omega}{2}, k_{m}^{\prime}=-\left(a+b+c+2 k_{m}\right) ; k_{m}^{\prime}$ und $k_{m}^{\prime \prime}$ sind also wieder zwei konjugierte Tripelpunkte.

5. Wir wollen nun die geometrische Bedeutung eines Tripelwendepunktes und eines Systems solcher Punkte festsetzen.

Ein Tripelwendepunkt ist ein Tripelpunkt, der mit seinem Tangentialpunkte zusammenfällt. Daraus ergibt sich, wenn $k_{w}$ ein solcher Tripelpunkt ist, die Bedingung: $k_{w}=-\left(a+b+c+2 k_{w}\right)+$ $+p \omega+p^{\prime} \omega^{\prime}$, wo $p$ und $p^{\prime}$ ganze Zahlen bedeuten; also $3 k_{\omega}=$ $=-(a+b+c)+p \omega+p^{\prime} \omega^{\prime}: k_{w}=-\frac{a+b+c}{3}+\frac{p \omega+p^{\prime} \omega^{\prime}}{3}$. Wir sehen, unser System enthält neun Tripelwendepunkte; wir erhalten sie, wenn wir in dem Ausdrucke für $k_{w} p$ und $p^{\prime}$ je $=0,1,2$ setzen, ganz wie bei dem Parameterausdruck für die gewöhnlichen Wendepunkte. Welche geometrische Eigenheit kommt nun einem solchen Tripelwendepunkte zu, z. B. dem Tripelwendepunkte $-\frac{a+b+c}{3}$, den wir aus dem Kollektivausdrucke für die Wendepunkte erhalten, wenn wir $p$ und $p^{\prime}=0$ setzen. Dieser Wendepunkt $-\frac{a+b+c}{3}$ wird gebildet von den drei Punkten, deren Parameter bezw. $a-\frac{a+b+c}{3}, b-\frac{a+b+c}{3}, c-\frac{a+b+c}{3}$ sind; es ist aber:

$a-\frac{a+b+c}{3}+b-\frac{a+b+c}{3}+c-\frac{a+b+c}{3} \equiv 0\left(\bmod \omega, \omega^{\prime}\right)$;

also: In dem System der Tripelpunkte sind diejenigen Tripelwendepunkte, deren drei Punkte in eine Gerade hineinfallen.

In der Tat fäilt ja ein solcher Tripelpunkt mit seinem Tangentialpunkte zusammen. 
Der Kollektivausdruck für die neun Tripelwendepunkte $k_{w}=\frac{-(a+b+c)+p \omega+p^{\prime} \omega^{\prime}}{3}$ läßt erkennen, daß man, wenn einer der Tripelpunkte, etwa $-\frac{a+b+c}{3}$, gegeben ist, in folgender Weise die anderen konstruieren kann: Projizieren wir die drei Punkte $a-\frac{a+b+c}{3}, b-\frac{a+b+c}{3}, c-\frac{a+b+c}{3}$ von einem Wendepunkte aus, z. B. dem Wendepunkte 0 , so erhalten wir $\frac{a+b+c}{3}-a, \frac{a+b+c}{3}-b, \frac{a+b+c}{3}-c$. Projizieren wir sodann diese drei Punkte von sämtlichen Wendepunkten aus, deren Parameter in dem Ausdrucke $\frac{p \omega+p^{\prime} \omega^{\prime}}{3}$ entbalten sind, so erhalten wir die sämtlichen Tripelwendepunkte

$$
\begin{gathered}
a-\frac{a+b+c}{3}+\frac{p \omega+p^{\prime} \omega^{\prime}}{3}, b-\frac{a+b+c}{3}+\frac{p \omega+p^{\prime} \omega^{\prime}}{3}, \\
c-\frac{a+b+c}{3}+\frac{p \omega+p^{\prime} \omega^{\prime}}{3} .
\end{gathered}
$$

Es ist selbstverständlich, daß die Sätze, die die gegenseitige Lage der eigentlichen Wendepunkte zum Gegenstande haben, sich ohne weiteres auf das System der Tripelwendepunkte übertragen lassen. Zwei derselben liegen z. B. immer auf einer Tripelgeraden, die noch einen dritten enthält. Das Bemerkenswerte dabei ist, dab eine beliebige Gerade $G$ der Ebene auf der $C_{3}$ die Punkte eines Tripelwendepunktes ausschneidet und so Veranlassung gibt zu einer Konfiguration, die der Wendepunktskonfiguration vollkommen analog ist.

6. Wir wollen drei in einer Geraden liegende Tripelwendepunkte betrachten, z. B.:

(1) $a-\frac{a+b+c}{3}, \quad b-\frac{a+b+c}{3}, \quad c-\frac{a+b+c}{3}$;

(2) $a-\frac{a+b+c}{3}-\frac{\omega}{3}, b-\frac{a+b+c}{3}-\frac{\omega}{3}, c-\frac{a+b+c}{3}-\frac{\omega}{3}$;

(3) $a-\frac{a+b+c}{3}-\frac{2 \omega}{3}, b-\frac{a+b+c}{3}-\frac{2 \omega}{3}, c-\frac{a+b+c}{3}-\frac{2 \omega}{3}$.

Die drei Punkte von (1) seien der Reihe nach die Punkte $A_{1}, B_{1}, C_{1}$; die von (2) $A_{2}, B_{2}, C_{2}$; die von (3) $A_{3}, B_{3}, C_{3}$. Dann liegen $A_{1}, B_{2}, C_{3}$ auf einer Geraden, ebenso $B_{1}, C_{2}, A_{3}$ und $C_{1}, A_{2}, B_{3}$, wie sich durch Addition. der Parameter unmittelbar ergibt; ebenso aber auch $A_{1}, C_{2}, B_{3} ; B_{1}, A_{2}, C_{3}$ und $C_{1}, B_{2}, A_{3}$. 
Der $C_{3}$ - Büschel $\left(A_{1} B_{1} C_{1} A_{2} B_{2} C_{2} A_{3} B_{3} C_{3}\right)$ enthält also drei Kurven, die in drei Gerade zerfallen.

Es ist noch bemerkenswert, daß ein Tripelpanktsystem $(a+k, b+k, c+k)$ ein eindeutig bestimmtes System von neun Wendepunkten

$$
\begin{gathered}
\left(a-\frac{a+b+c+p \omega+p^{\prime} \omega^{\prime}}{3}, b-\frac{a+b+c+p \omega+p^{\prime} \omega^{\prime}}{3},\right. \\
\left.c-\frac{a+b+c+p \omega+p^{\prime} \omega^{\prime}}{3}\right)
\end{gathered}
$$

hat, ganz unabbängig von der Wahl des Ausgangstripelpunktes innerhalb des Systems. Setzen wir z. B. $a+k=a^{\prime}, b+k=b^{\prime}$, $c+k=c^{\prime}$ und denken uns unsere Dreieckmannigfaltigkeit durch die drei Parameter $a^{\prime}+s, b^{\prime}+s, c^{\prime}+s$ gegeben, wobei $s$ variabel ist, nehmen also $a^{\prime}, b^{\prime}, c^{\prime}$ als den Ausgangstripelpunkt, so ist das System der neun Wendetripelpunkte gegeben durch die Parameterausdrü.cke

$$
\begin{gathered}
a^{\prime}-\frac{a^{\prime}+b^{\prime}+c^{\prime}+p \omega+p^{\prime} \omega^{\prime}}{3}, \quad b-\frac{a^{\prime}+b^{\prime}+c^{\prime}+p \omega+p^{\prime} \omega^{\prime}}{3}, \\
c^{\prime}-\frac{a^{\prime}+b^{\prime}+c^{\prime}+p \omega+p^{\prime} \omega^{\prime}}{3}
\end{gathered}
$$

drücken wir nun aber $a^{\prime}, b^{\prime}, c^{\prime}$ durch $a, b, c$ aus, so gehen diese Ausdrücke über in:

$$
\begin{aligned}
& a+k-\frac{a+k+b+k+c+k+p \omega+p^{\prime} \omega^{\prime}}{3}=a-\frac{a+b+c+p \omega+p^{\prime} \omega^{\prime}}{3} \\
& b+k-\frac{a+k+b+k+c+k+p \omega+p^{\prime} \omega^{\prime}}{3}=b-\frac{a+b+c+p \omega+p^{\prime} \omega^{\prime}}{3} \\
& c+k-\frac{a+k+b+k+c+k+p \omega+p^{\prime} \omega^{\prime}}{3}=c-\frac{a+b+c+p \omega+p^{\prime} \omega^{\prime}}{3},
\end{aligned}
$$

womit unsere Behauptung bewiesen ist.

7. Spezielle Fälle. Nehmen wir an Stelle der drei Ausgangspunkte $a, b, c$ drei unendlich benachbarte Punkte an, so ergibt sich ein Tripelpunktsystem, das mit dem gewöhnlichen Punktsystem identisch ist; in der Tat ergeben sich aus unserem Ausdrucke für die Tripelwendepunkte die gewöhnlichen Wendepunkte, wenn wir $a=b=c$ setzen. Das System der einfachen Punkte der $C_{3}$ ist also als spezieller Fall in unserer zweifach unendlichen Mannigfaltigkeit von Tripelpunktsystemen enthalten - dab diese Mannigfaltigkeit zweifach unendiich ist, kann man am einfachsten an der schon erwähnten Tatsache erkennen, daß jede Gerade der Ebene einen Tripelwendepunkt eines solchen Systems liefert. 
Bemerkenswert sind noch vier andere Tripelpunktsysteme, die Systeme der sogenannten Punkttripel, die ich bei verschiedenen Gelegenheiten "merkwürdige Dreiecke" genannt habe; es sind dies die Systeme $\left(k, \frac{\omega}{3}+k, \frac{2 \omega}{3}+k\right),\left(k, \frac{\omega^{\prime}}{3}+k, \frac{2 \omega^{\prime}}{3}+k\right)$, $\left(k, \frac{\omega+\omega^{\prime}}{3}+k, \frac{2 \omega+2 \omega^{\prime}}{3}+k\right),\left(k, \frac{\omega+2 \omega^{\prime}}{3}+k_{3} \frac{2 \omega+\omega^{\prime}}{3}+k\right)$, wobei $k$ der veränderliche Parameter ist. . Sie sind dadurch gekennzeichnet, daßs sie Tripelpunkte enthalten, die aus drei in einer Geraden liegenden einfachen Wendepunkten bestehen; wir haben für jedes der vier Systeme denjenigen Tripelwendepunkt als Ausgangspunkt gewählt, der den Wendepunkt 0 enthält. Ein Dreieck eines solchen Systems - $k, \frac{\omega}{3}+k, \frac{2 \omega}{3}+k$ - hat bekanntlich die Eigenschaft, daß der dritte Schnittpunkt einer Seite zugleich der Tangentialpunkt des dritten Eckpunktes ist: Der dritte Schnittpunkt der Geraden $\left(k, \frac{\omega}{3}+k\right)$ z. B. ist $-2 k-\frac{\omega}{3}$ und der Tangentialpunkt des dritten Eckpunktes $\frac{2 \omega}{3}+k$ ist $=-2 k-$ $-\frac{4 \omega}{3} \equiv-2 k-\frac{\omega}{3}(\bmod \omega)$, also derselbe Punkt. Man kann also zu einem Tripelpunkt entweder auf die gewöhnliche Weise, durch Bestimmung der dritten Schnittpunkte der Seiten, den Tangentialpunkt bestimmen oder indem man zu den drei Eckpunkten ibre Tangentialpunkte zeichnet. Diese doppelte Möglichkeit der Konstruktion eines Tangentialtripelpunktes ist nur eine Konsequenz der Lage von drei in einer beliebigen Tripelgeraden liegenden Tripelpunkten. Sind $a_{1} b_{1} c_{1}, a_{2} b_{2} c_{2}, a_{3} b_{3} c_{3}$ drei in einer solchen Geraden liegende Tripelpunkte unseres Systems $\left(k, \frac{\omega}{3}+k, \frac{2 \omega}{3}+k\right)$, so sind immer zwei von ihnen dreifach perspektivisch, derart, dah die Eckpunkte des dritten die zugehörigen Projektionszentren sind. Es existieren nämlich außer den Verbindungslinien, die bei den Punkten jeder Tripelgeraden vorhanden sind, noch die Geraden $a_{1} a_{2} a_{3}, b_{1} b_{2} b_{3}, c_{1} c_{2} c_{3}$. Man erkennt das sofort, wenn man die Dreiecke durch die Parameter ausdrückt:

die von $a_{1}, a_{2}, a_{3}$ seien bezw. $k_{1}, \frac{\omega}{3}+k_{1}, \frac{2 \omega}{3}+k_{1}$

$$
\begin{gathered}
n \quad b_{1}, b_{2}, b_{3} \quad " \quad k_{2}, \frac{\omega}{3}+k_{2}, \quad \frac{2 \omega}{3}+k_{2} ; \text { dann sind } \\
" \quad " \quad c_{1}, c_{2}, c_{3}-\left(k_{1}+k_{2}\right), \frac{\omega}{3}-\left(k_{1}+k_{2}\right), \frac{2 \omega}{3}-\left(k_{1}+k_{2}\right) .
\end{gathered}
$$


Es ist alsdann $\quad k_{1}+k_{2}-\left(k_{1}+k_{2}\right) \equiv 0$

$$
\begin{aligned}
\frac{\omega}{3}+k_{1}+\frac{\omega}{3}+k_{2}+\frac{\omega}{3}-\left(k_{1}+k_{2}\right) & \equiv 0 \\
\frac{2 \omega}{3}+k_{1}+\frac{2 \omega}{3}+k_{2}+\frac{2 \omega}{3}-\left(k_{1}+k_{2}\right) & \equiv 0 .
\end{aligned}
$$

Anmerkung. Bei einem beliebigen Ausgangstripelpunkt $a b c$ ist $\mathrm{ja}$, wenn $k_{1}, k_{2}, k_{3}$ einer Tripelgeraden angehören, $k_{3} \equiv-\left(a+b+c+k_{1}+k_{2}\right) ; \quad$ in unserem Falle ist aber $a+b+c=0+\frac{\omega}{3}+\frac{2 \omega}{3} \equiv 0(\bmod \omega)$. -

Der Umstand nun, daß einerseits die Verbindungslinie $a_{1} a_{2}$ zu dem dritten einfachen Punkt $a_{3}$ führt, anderseits die Linien $a_{1} a_{2}, b_{1} b_{2}, c_{1} c_{2}$ durch ihre dritten Schnittpunkte den Tripelpunkt $a_{3} b_{3} c_{3}$ liefern, von dem derselbe einfache Punkt $a_{3}$ auch wieder ein Eckpunkt ist, läßt sich in folgendem Satze aussprechen:

Dieselben Punktgruppen, a uf die die Sätze der $C_{3}$ sich unmittelbar beziehen, sind zugleich Teile von gröberen Punktgrappen, auf die dieselben Sätze Anwendung finden, aber bei der übertragenen Bedeutung der Bezeichnungen "Punkt" und "Gerade als "Tripelpunkt" und "Tripelgerade".

Ich will zwei kleine Beispiele dafür geben.

1. Sind $a_{11}, a_{21}$ und $b_{11}, b_{21}$ zwei konjugierte Punktpaare desselben Systems, so liefern die Geraden $a_{11} b_{11}$ und $a_{21} b_{11}$ ein weiteres konjugiertes Punktpaar $c_{11} c_{21}$, dasselbe, das auch die Geraden $a_{11} b_{21}$ und $a_{21} b_{21}$ liefern. Sind aber diese Punkte $a_{11}, a_{21}$, $b_{11}, b_{21}$ Eckpunkte von Tripelpunkten unseres Systems: 1. $a_{11} a_{12} a_{13}$; 2. $a_{21} a_{22} a_{23}$; 3. $b_{11} b_{12} b_{13} ; 4 . b_{21} b_{22} b_{23}$, so sind 1 und 2 , 3 und 4 auch konjugierte Tripelpunktpaare und die Verbindungsinien 1,3 und 1, 4 liefern, ebenso wie die Verbindungslinien 2, 3 und 2,4 das weitere konjugierte Punktpaar $c_{11} c_{12} c_{13}$ und $c_{21} c_{22} c_{33}$, von dem die einfachen konjugierten Punkte $c_{11}$ und $c_{21}$ wieder Bestandteile sind.

2. Ist $a_{11}, a_{21}, a_{31}, a_{41}$ ein Quadrupel von Berührungspunkten von vier von einem Punkte $b_{1}$ der $C_{3}$ ausgehenden Tangenten, so liegen bekanntlich diese vier Berührungspunkte auf einer $C_{2}$, die die $C_{3}$ in $b_{1}$ berührt. Zugleich sind aber diese fünf Punkte $a_{11}, a_{21}, a_{31}, a_{41}, b_{1}$ Eckpunkte von fünf Tripelpankten unseres Systems $a_{11} a_{12} a_{13}, a_{21} a_{22} a_{23}, \ldots b_{1} b_{2} b_{3}$, kurz mit $a_{11} \ldots, a_{21} \ldots$, $a_{31} \ldots, a_{41} \ldots, b_{1} \ldots$ bezeichnet, es ist nun auch $b_{1} \ldots$ der Tangentialpunkt der vier erstgenannten Tripelpunkte, ebenso wie $b_{1}$ der Tangentialpunkt von $\alpha_{11}, a_{12}, a_{13}, a_{14}$ ist. Es liegen ferner auch die vier Tripelpunkte $a_{11} \ldots, a_{21} \ldots, a_{31} \ldots, a_{41} \ldots$ auf einem Tripelkegelschnitt, der außerdem noch die $C_{3}$ in den drei Punkten des 
Tangentialtripelpunktes $b_{1} \ldots$ berührt. - Daß der Tripelkegelschnitt eine $C_{6}$ ist, werden wir in (8) sehen. Also:

$$
a_{11}, a_{21}, a_{31}, a_{41}, b_{1}, b_{1}
$$

liegen auf einem Kegelschnitte;

$$
a_{11} \ldots, a_{21} \ldots, a_{31} \ldots, a_{41} \ldots, b_{1} \ldots, b_{1} \ldots
$$

liegen auf einem Tripelkegelschnitte.

Unsere vier merkwürdigen Systeme zeichnen sich aber auch noch in anderer Beziehung aus. Nehmen wir das System $\left(k, \frac{\omega}{3}+k, \frac{2 \omega}{3}+k\right)$, das einzige reelle von den vieren, und bestimmen seine Tripelwendepunkte, so ist, wie man unmittelbar erkennit, ein Tripelwendepunkt $o, \frac{\omega}{3}, \frac{2 \omega}{3}$; ein zweiter ist

$$
\frac{\omega^{\prime}}{3}, \frac{\omega}{3}+\frac{\omega^{\prime}}{3}, \frac{2 \omega}{3}+\frac{\omega^{\prime}}{3} ; \text { ein dritter } \frac{2 \omega^{\prime}}{3}, \frac{\omega}{3}+\frac{2 \omega^{\prime}}{3}, \frac{2 \omega}{3}+\frac{2 \omega^{\prime}}{3} .
$$

Auf diese drei scheint sich diesmal die Zahl der Tripelwendepunkte zu beschränken; dem ist aber in Wirklichkeit nicht so. Um das zu erkennen, müssen wir noch einmal auf die Natur eines Tripelwendepunktes eingehen. Als einen solchen erkannten wir einen Tripelpunkt, dessen drei Punkte in eine Gerade fallen. Offenbar muß aber auch ein solches Dreieck $a b c$ als ein Tripelwendepunkt angesehen werden, dessen drei Seiten $a b, b c, c a$ die $C_{3}$ berühren; denn bei einem solchen Dreieck fallen ja die dritten Schnittpunkte der Seiten mit den Eckpunkten zusammen; ein solcher Tripelpunkt $a b c$ ist also auch sein eigener Tangentialpunkt. Dieser Fall eines Tripelwendepunktes brauchte bisher nicht erörtert za werden, da ein beliebiges Tripelpunktsystem keinen solchen Wendepunkt besitzt. Die 24 um- und einbeschriebenen Dreiecke gehören vielmehr ausschließlich den vier ausgezeichneten Systemen an; jedes besitzt deren sechs. Zum Beispiel hat das reelle System folgende um- and einbeschriebene Dreiecke als weitere Tripelwendepunkte:

$$
\text { a) } \frac{\omega}{9}, \frac{\omega}{3}+\frac{\omega}{9}, \frac{2 \omega}{3}+\frac{\omega}{9}
$$

oder umgestellt :

$$
\begin{aligned}
& \frac{\omega}{9}, \frac{2 \omega}{3}+\frac{\omega}{9}, \frac{\omega}{3}+\frac{\omega}{9} \equiv \frac{\omega}{9}, \frac{-2 \omega}{9}, \frac{+4 \omega}{9} . \\
& \text { b) } \frac{2 \omega}{9}, \frac{\omega}{3}+\frac{2 \omega}{9}, \frac{2 \omega}{3}+\frac{2 \omega}{9} \equiv \frac{2 \omega}{9}, \frac{-4 \omega}{9}, \frac{8 \omega}{9}, \\
& \text { c) } \frac{\omega^{\prime}}{3}+\frac{\omega}{9}, \frac{\omega^{\prime}}{3}+\frac{4 \omega}{9}, \frac{\omega^{\prime}}{3}+\frac{7 \omega}{9},
\end{aligned}
$$


oder umgestellt:

$\frac{\omega^{\prime}}{3}+\frac{\omega}{9}, \frac{\omega^{\prime}}{3}+\frac{7 \omega}{9}, \frac{\omega}{3}+\frac{4 \omega}{9} \equiv \frac{\omega^{\prime}}{3}+\frac{\omega}{9}, \frac{-2 \omega^{\prime}}{3}-\frac{2 \omega}{9}, \frac{+4 \omega^{\prime}}{3}+\frac{4 \omega}{9}$.

d) $\frac{\omega^{\prime}}{3}+\frac{2 \omega}{9}, \frac{\omega^{\prime}}{3}+\frac{5 \omega}{9} \equiv-\frac{2 \omega^{\prime}}{3}-\frac{4 \omega}{9}, \frac{\omega^{\prime}}{3}+\frac{8 \omega}{9}$.

e) $\frac{2 \omega^{\prime}}{3}+\frac{\omega}{9}, \frac{2 \omega^{\prime}}{3}+\frac{4 \omega}{9}, \frac{2 \omega^{\prime}}{3}+\frac{7 \omega}{9}$,

oder umgestellt:

$$
\begin{gathered}
\frac{2 \omega^{\prime}}{3}+\frac{\omega}{9}, \frac{2 \omega^{\prime}}{3}+\frac{7 \omega}{9} \equiv-\frac{4 \omega^{\prime}}{3}-\frac{2 \omega}{9}, \frac{2 \omega^{\prime}}{3}+\frac{4 \omega}{9} . \\
\text { f) } \frac{2 \omega^{\prime}}{3}+\frac{2 \omega}{9}, \frac{2 \omega^{\prime}}{3}+\frac{5 \omega}{9} \equiv-\frac{4 \omega^{\prime}}{3}-\frac{4 \omega}{9}, \frac{2 \omega^{\prime}}{3}+\frac{8 \omega}{9} .
\end{gathered}
$$

Nur die ersten zwei dieser sechs Tripelwendepunkte sind reell.

Im ganzen hat dieses System also auch wieder drei reelle Tripelwendepunkte: $a$ ) und $b$ ) und den Tripelwendepunkt $0, \frac{\omega}{3}, \frac{2 \omega}{3}$. Die Konfiguration der neun Tripelwendepunkte ist im übrigen bei diesen vier Systemen genau dieselbe wie bei jedem anderen System.

Ich möchte schließlich noch ein weiteres spezielles System erwähnen, das System $\left(a+k, a+\frac{\omega}{2}+k, a+\frac{\omega^{\prime}}{2}+k\right)$ mit $k$ als veränderlichem Parameter. Ein Tripelpunkt dieses Systems hat die Eigenschaft, daß zwei seiner Punkte untereinander und dem dritten konjugiert sind. Die neun Tripelwendepunkte dieses Systems werden von den neun harmonischen Polaren der $C_{3}$ gebildet; diese veranlassen also ebenfalls eine Wendepunktskonfiguration.

8. Wir kehren zur allgemeinen Tripelpunkttheorie zurück. Wir haben den Tripelpunkt und die Tripelgerade in bestimmter Weise definiert; da erhebt sich nun die Frage: ist durch diese Definitionen ein bestimmter geometrischer Sinn auch für Bezeichnungen, wie Tripelkegelschnitt, ... Tripel- $C_{n}$ festgelegt? Daß dieses der Fall ist und in welcher Weise, läßt sich leicht erkennen.

Sind $k_{1}, k_{2}, k_{3}, \ldots k_{6}$ die Parameter von sechs Punkten eines Kegelschnittes, so ist bekanntlich $k_{1}+k_{2}+\cdots+k_{6} \equiv 0(\bmod \omega, \omega)$; sollen nun diese Parameter Dreiecke bedeuten, so ist die analoge Beziehung damit gegeben, daß

also

$$
\begin{aligned}
& \text { 1. } a+k_{1}+b+k_{1}+c+k_{1}+a+k_{2}+\cdots \\
& +a+k_{6}+b+k_{6}+c+k_{6} \equiv 0\left(\bmod \omega, \omega^{\prime}\right) \text {; }
\end{aligned}
$$

$$
6(a+b+c) \equiv-3\left(k_{1}+k_{2}+\cdots+k_{6}\right)
$$


oder

2) $k_{1}+k_{2}+\cdots+k_{0} \equiv-2(a+b+c)\left(\bmod \omega, \omega^{\prime}\right)$.

Die Gleichung 1) drückt aber aus, daß die sechs Tripelpunkte $k_{1} \ldots k_{6}$ auf einer $C_{6}$ liegen; das gleiche besagt also auch die aus 1) sich ergebende Gleichung 2) und allgemein drückt die Gleichung 3):

$$
k_{1}+k_{2}+\cdots+k_{3 n} \equiv-n(a+b+c)
$$

aus, daß die $3 n$ zu diesen Parametern gehörigen Tripelpunkte auf einer $C_{n}$ liegen. Der Tripelkegelsehnitt ist also eine $C_{6}$, die Tripel $C_{n}$ eine $C_{3 n}$.

Diese Erkenntnis setzt uns in den Stand, weitere bemerkenswerte Analogien zur Theorie der einfachen Punkte aufzustellen.

Ist' z. B.

$$
k_{1}+k_{2}+\cdots+k_{p}+k_{p+1}+\cdots+k_{3 n} \equiv-n(a+b+c)
$$

und ebenso

$$
k_{1}+k_{2}+\cdots+k_{p}+k_{p+1}^{\prime}+\cdots+k_{3 n}^{\prime} \equiv-n(a+b+c),
$$

dann ist offenbar

$$
k_{p+1}+\cdots+k_{3 n} \equiv k_{p+1}^{\prime}+\cdots+k_{3 n}^{\prime}
$$

jede Summe von $p$ Parametern also, die $k_{p+1}^{\prime}+\cdots+k_{3 n}^{\prime}$ zu der Gröbe $A=-n(a+b+c)+p \omega+p^{\prime} \omega^{\prime}$ ergänzt, ergänzt auch die Summe $k_{p+1}^{\prime}+\cdots+k_{3 n}^{\prime}$ zu einer Größe, die sich von $A$ nur um ein vielfaches der Periodizitätsmoduln unterscheiden kann; d. h. die Tripelpunktgruppen $k_{p+1}, k_{p+2}, \cdots k_{3 n}$ und $k_{p+1}^{\prime}, k_{p+2}^{\prime}, \cdots k_{3 n}^{\prime}$ sind korresidual.

Ich will nun noch einige Anwendungen von der Gleichung 3$)$ : $k_{1}+k_{2}+\cdots+k_{3 n}=-n(a+b+c)$ geben.

a) Die harmonische Tripelpolare.

Wir haben im Abschnitt 3. zu dem Tangentialtripelpunkte $k^{\prime}$ das Quadrupel der Berührungspunkte der vier weiteren von diesem Punkt ausgehenden Tangenten bestimmt und dafür den Ausdruck gefunden: $x=-\frac{a+b+c}{2}+\frac{p \omega+p^{\prime} \omega^{\prime}}{2}-\frac{k^{\prime}}{2}$. Ist nun $k^{\prime}$ ein Tripelwendepunkt, z. B. $=-\frac{a+b+c}{3}$, so erhalten wir:

$$
\begin{gathered}
x=-\frac{a+b+c}{2}+\frac{p \omega+p^{\prime} \omega^{\prime}}{2}+\frac{a+b+c}{6}=-\frac{a+b+c}{3}+ \\
+\frac{p \omega-p^{\prime} \omega^{\prime}}{2}
\end{gathered}
$$


$x_{1}=-\frac{a+b+c}{3}$ fällt mit dem Tripelwendepunkte selbst zusammen.

$x_{2}=-\frac{a+b+c}{3}+\frac{w}{2}$

$x_{3}=-\frac{a+b+c}{3}+\frac{\omega^{\prime}}{2}$

$x_{4}=-\frac{a+b+c}{3}+\frac{\omega+\omega^{\prime}}{2}$

$x_{2}+x_{3}+x_{4}=-(a+b+c) ; d$. h. die drei Berührungstripelpunkte liegen auf einer Geraden, der, ,harmonischen Tripelpolare" zu dem Tripelwendepunkte $-\frac{a+b+c}{3}$.

b) Irgend eine Gerade $w p q$ durch den Wendepunkt $w$ schneidet bekanntlich auf der $C_{3}$ zwei Punkte $p$ und $q$ von der Eigenschaft aus, da $\beta$ ein Kegelschnitt die $C_{3}$ in $p$ und $q$ je dreipunktig berïhrt; fallen die beiden Punkte in einen Punkt $r$ der harmonischen Polare zusammen, so berührt eine $C_{2}$ die $C_{3}$ in $r$ sechspunktig.

Wir wollen diesen Satz auf unser Tripelpunktsystem uibertragen. Der Tripelwendepunkt $w$ habe den Parameter $-\frac{a+b+c}{3}$; irgend eine durch ihn gelegte Tripelgerade schneide einen Tripelpunkt mit dem Parameter $p$ aus; der dritte Schnittpunkt ist dann $q=-(a+b+c)-p+\frac{a+b+c}{3}=-\frac{2}{3}(a+b+c)-p . \quad \mathrm{Es}$ gibt alsdann eine $C_{6}$, die in den drei Punkten des Tripelpunktes $p$ und in denen von $q$ je dreifach berührt; denn es ist

$$
3 p+3 \cdot\left[-\frac{2}{3}(a+b+c)-p\right]=-2(a+b+c) \text {. }
$$

Lassen wir nun $p$ und $q$ in einen Tripelpunkt $r$ der harmonischen Tripelpolare zusammenfallen, so ist

$6 \cdot\left(-\frac{a+b+c}{3}+\frac{p \omega+p^{\prime} \omega^{\prime}}{2}\right) \equiv-2(a+b+c)\left(\bmod \omega, \omega^{\prime}\right)$, woraus ersichtlich ist, daß tatsächlich jeder dieser Tripelpunkte $--\frac{a+b+c}{3}+\frac{p \omega+p^{\prime} \omega^{\prime}}{2}-$ mit seinen drei je sechsfach zu zählenden Punkten 18 Punkte ergibt, die die Schnittpunkte einer $C_{6}$ mit der $C_{3}$ sind.

10. Wir haben bisher der Lage des Ausgangsdreieckes $a, b, c$ keine Beachtung geschenkt. Es läßt sich nun aber zeigen, dab bei entsprechender Auswahl dieses Dreieckes unsere Parametergleichungen sich einfacher gestalten. Wählen wir nämlich als solches einen Tripelwendepunkt, z. B. 


$$
a^{\prime}=a-\frac{a+b+c}{3}, b^{\prime}=b-\frac{a+b+c}{3}, c^{\prime}=c-\frac{a+b+c}{3},
$$

so ist

$$
a^{\prime}+b^{\prime}+c^{\prime} \equiv o\left(\bmod w, w^{\prime}\right)
$$

in diesem Falle stimmen also die Parametergleichungen der Tripelpunkte mit den Parametergleichungen der einfachen Punkte überein, da ja in den ersteren die rechte Seite immer ein Vielfaches von $-(a+b+c)$ ist. Diese Übereinstimmung läht eine bemerkenswerte geometrische Deutung zu. Betrachten wir den Tripelpunkt $a-\frac{a+b+c}{3}+k=a^{\prime}+k, \quad b-\frac{a+b+c}{3}+k=b^{\prime}+k$, $c-\frac{a+b+c}{3}+k=c^{\prime}+k$, so ist $a^{\prime}+k$ offenbar der dritte Schnittpunkt der Verbindungslinie der zwei Punkte $-a^{\prime}$ und $-k$, $b^{\prime}+k$, ebenso der dritte Schnittpunkt der Geraden $\left(-b^{\prime},-k\right)$, $c^{\prime}+k$, der dritte Schnittpunkt der Geraden $\left(-c^{\prime},-k\right)$; d. h. der Tripelpunkt $a^{\prime}+k, b^{\prime}+k, a^{\prime}+k$ wird erhalten als Projektion des Tripelwendepunktes $\left(-a^{\prime},-b^{\prime},-c^{\prime}\right)$ von dem Punkte - $k$ aus. Dieser Tripelwendepunkt gehört dem System an, das man erhält, wenn man sämtliche Tripelpunkte des gegebenen Systems $(a+k$, $b+k, c+k)$ von dem Wendepunkte 0 aus projiziert. Wir erbalten somit unser Tripelpunktsystem, wenn wir diesen Tripelwendepunkt $\left(-a+\frac{a+b+c}{3},-b+\frac{a+b+c}{3},-c+\frac{a+b+c}{3}\right)$ von sämtlichen Punkten $-k$ der $C_{3}$ aus projizieren. Durch dieses Arrangement wird also zwischen unserem Tripelpunktsystem und dem System der einfachen Punkte der $C_{3}$ eine perspektivische Beziehung: hergestellt: Die drei Punkte eines Tripelpunktes $k$ ergeben sich als die Projektion der drei in einer Geraden liegenden Punkte $-a+\frac{a+b+c}{3},-b+\frac{a+b+c}{3},-c+\frac{a+b+c}{3} ;$ das zugehörige Projektionszentrum $-k$ ist der dem Tripelpunkte $k$ entsprechende Punkt. Liegen die drei einfachen Punkte $-k_{1},-k_{2}$, $-k_{3}$ auf einer Geraden, dann bilden die zugehörigen Tripelpunkte $k_{1}, k_{2}, k_{3}$ eine Tripelgerade; ist $k_{1}=k_{2}$ und $-k_{3}$ der Tangentialpunkt von $-k_{1}$, dann ist auch der zugehörige Tripelpunkt $k_{3}$ der Tangentialpunkt des Tripelpunktes $k_{1}$ u. s. w.

11. Ich will nun die Beschaffenheit einer Tripelgeraden näher untersuchen. Eine solche Tripelgerade wird offenbar von einem Büschel von $C_{3}$ veranlabt, der zwei in je drei Geraden zerfallende Kurven hat, $l_{1} l_{2} l_{3}$ und $m_{1} m_{2} m_{3}$. Fig. 1 (s. folgende Seite) enthält drei in einer Tripelgeraden liegende Tripelpunkte:

$$
\text { a) } \begin{array}{lll}
a+k_{1} & b+k_{1} & c+k_{1} \\
a+k_{2} & b+k_{2} & c+k_{2} \\
a+k_{3} & b+k_{3} & c+k_{8}
\end{array}
$$


jede der drei wagrechten Zeilen von $\alpha$ ) gibt die drei einfachen Punkte, die zu einem Tripelpunkte sich zusammensetzen. Daßb die drei Tripelpunkte auf einer Tripelgeraden liegen, ergibt sich daraus, daf folgende Geraden vorhanden sind (s. Fig. 1): $a+k_{1} b+k_{2} c+k_{3}$ and $a+k_{1} b+k_{3} c+k_{2} ; b+k_{1} a+k_{2} c+k_{3}$ und $b+k_{1} c+k_{2}$ $a+k_{3} ; c+k_{1} a+k_{2} b+k_{3}$ und $c+k_{1} a+k_{3} b+k_{2}$ (s. $\S 1$.).

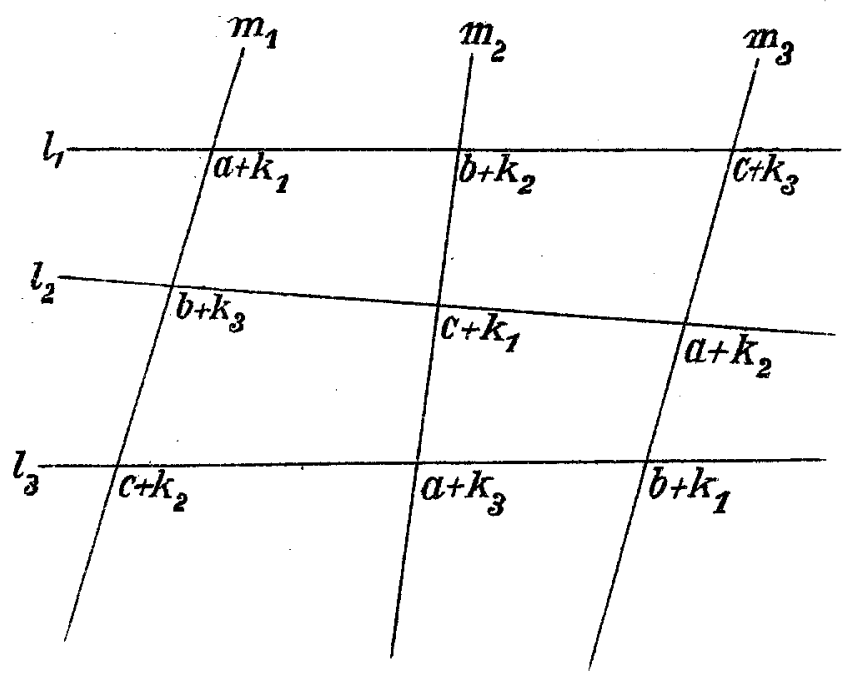

Fig. 1.

Bestimmen wir nun aber dieselben Geraden anstatt aus der Zeichnung aus dem System $\alpha$ ), so springt beim Ablesen dieser Geraden die vollkommene Analogie mit der Auswertung einer Determinante in die Augen. Nun können bei der Berechnung einer Determinante die Vertikalreihen in gleicher Weise benützt werden wie die Horizontalreihen. Es ist daher zu vermuten, daß vielleicht auch die Vertikalreihen Tripelpunkte einer Tripelgeraden sind. In der Tat sieht man sofort, dalo auch $a+k_{1} a+k_{2} a+k_{3}$, $b+k_{1} b+k_{2} b+k_{3}, c+k_{1} c+k_{2} c+k_{3}$ Tripelpunkte eines Tripelpunktsystems sind: Der Ausgangstripelpunkt ist $k_{1} k_{2} k_{3}$, die Parameter der drei Tripelpunkte sind bezw. $a, b, c$. Wir wollen die zwei Geraden, die in $\alpha$ ) enthalten sind, zwei konjugierte Gerade nennen und kommen so zu dem Satze:

Zu jeder Tripelpunktgeraden gehört eine konjugierte Gerade.

12. Fortsetzung. Die weiteren Betrachtungen, die sich hier anstellen lassen, knüpfen sich an die Wendepunkte der beiden Tripelpunktsysteme. Die Wendepunkte des ersten Systems mit dem Ausgangstripelpunkte $(a, b, c)$ sind gegeben durch 


$$
\text { B) }-\frac{a+b+c}{3}+\frac{p \omega+p^{\prime} \omega^{\prime}}{3} \text {; }
$$

die des zweiten Systems mit dem Ausgangspunkte $\left(k_{1}, k_{2} ; k_{3}\right)$ durch

$$
\gamma)-\frac{k_{1}+k_{2}+k_{3}}{3}+\frac{p \omega+p^{\prime} \omega^{\prime}}{3} \text {. }
$$

also

Nun ist (s. Fig. 1) $a+k_{1}+b+k_{2}+c+k_{3} \equiv 0\left(\bmod \omega, \omega^{\prime}\right)$;

$$
\begin{aligned}
& k_{1}+k_{2}+k_{3}=-(a+b+c)+p \omega+p^{\prime} \omega^{\prime} ; \\
& \frac{k_{1}+k_{2}+k_{3}}{3}=-\frac{a+b+c}{3}+\frac{p \omega+p^{\prime} \omega^{\prime}}{3} ;
\end{aligned}
$$

folglich kann $\gamma$ ) ersetzt werden durch $\frac{a+b+c}{3}+\frac{p \omega+p^{\prime} \omega^{\prime}}{3}$.

Die Wendepunkte des ersten Systems sind also:

$$
\text { ع) }\left\{\begin{array}{l}
a-\frac{a+b+c+p \omega+p^{\prime} \omega^{\prime}}{3} \\
b-\frac{a+b+c+p \omega+p^{\prime} \omega^{\prime}}{3} \\
c-\frac{a+b+c+p \omega+p^{\prime} \omega^{\prime}}{3}
\end{array}\right.
$$

die des zweiten Systems:

$$
\left.\varepsilon^{\prime}\right)\left\{\begin{array}{l}
k_{1}+\frac{a+b+c+p \omega+p^{\prime} \omega^{\prime}}{3} \\
k_{2}+\frac{a+b+c+p \omega+p^{\prime} \omega^{\prime}}{3} \\
k_{3}+\frac{a+b+c+p \omega+p^{\prime} \omega^{\prime}}{3}
\end{array} .\right.
$$

Projizieren wir diese zwei Tripelwendepunktsysteme von dem Wendepunkte 0 aus, so erbalten wir die Wendepunktsysteme, die. zu den Tripelpunktsystemen $(-a,-b,-c)$ und $\left(-k_{1},-k_{2},-k_{3}\right)$ gehören. Sie sind:

$$
\text { ९) }\left\{\begin{array}{l}
-a+\frac{a+b+c+p \omega+p^{\prime} \omega^{\prime}}{3} \\
-b+\frac{a+b+c+p \omega+p^{\prime} \omega^{\prime}}{3} \\
-c+\frac{a+b+c+p \omega+p^{\prime} \omega^{\prime}}{3}
\end{array}\right.
$$




$$
\left.\varphi^{\prime}\right)\left\{\begin{array}{l}
-k_{1}-\frac{a+b+c+p \omega+p^{\prime} \omega^{\prime}}{3} \\
-k_{2}-\frac{a+b+c+p \omega+p^{\prime} \omega^{\prime}}{3} \\
-k_{3}-\frac{a+b+c+p \omega+p^{\prime} \omega^{\prime}}{3}
\end{array}\right.
$$

Bilden wir nun die dritten Schnittpunkte der Verbindungslinien aller Punkte $\varphi$ ) mit allen Punkten $\varphi^{\prime}$ ), so erhalten wir das System von Punkten

$g)\left\{\begin{array}{l}a+k_{1}+\frac{p \omega+p^{\prime} \omega^{\prime}}{3} ; b+k_{1}+\frac{p \omega+p^{\prime} \omega^{\prime}}{3}, c+k_{1}+\frac{p \omega+p^{\prime} \omega^{\prime}}{3} \\ a+k_{2}+\frac{p \omega+p^{\prime} \omega^{\prime}}{3}, b+k_{2}+\frac{p \omega+p^{\prime} \omega^{\prime}}{3}, c+k_{2}+\frac{p \omega+p^{\prime} \omega^{\prime}}{3} \\ a+k_{3}+\frac{p \omega+p^{\prime} \omega^{\prime}}{3}, b+k_{3}+\frac{p \omega+p^{\prime} \omega^{\prime}}{3}, c+k_{3}+\frac{p \omega+p^{\prime} \omega^{\prime}}{3} .\end{array}\right.$

Das sind aber die Punkte unserer Anfangstripelgeraden und der acht weiteren Geraden, die sich ergeben, wenn wir die gegebene Tripelgerade von dem Wendepunite 0 aus projizieren und die Projektion von den acht weiteren Wendepunkten aus wiederum projizieren.

13. Bevor ich dieses Ergebnis in einem Satze formuliere, mub ich eine Betrachtung über Tripelpunktsysteme zweiten Grades anstellen. Gerade so, wie wir irgend drei einfache Punkte $a, b, c$ zur Herstellung des Tripelpunktsystems $(a, b, c)$ benützt haben, können wir drei Tripelpunkte herausgreifen und daraus ein Tripelpunktsystem zweiten Grades bilden. Ein Tripelpunkt zweiten Grades setzt sich also aus drei Dreiecken zusammen:

$$
\text { a) }\left\{\begin{array}{lll}
a+h_{1} & b+h_{1} & c+h_{1} \\
a+h_{2} & b+h_{2} & c+h_{2} \\
a+h_{3} & b+h_{3} & c+h_{3} .
\end{array}\right.
$$

Um nun aus diesen drei beliebigen Tripelpunkten des Systems $(a+h, b+h, c+-h)$ das durch sie bestimmte Tripelpunktsystem zweiten Grades herzustellen, verfahren wir wie bei der Bildung eines Tripelpunktsystems ersten Grades: wir projizieren den Tripelpunkt zweiten Grades a) von irgend einem Tripelpunkt ersten Grades

$$
a+m \quad b+m \quad c+m \quad \text { aus. }
$$


Wir erhalten so

$$
\text { 3) }\left\{\begin{array}{c}
a-\left(a+b+c+h_{1}+m\right) \quad b-\left(a+b+c+h_{1}+m\right) \\
c-\left(a+b+c+h_{1}+m\right) \\
a-\left(a+b+c+h_{2}+m\right) \quad b-\left(a+b+c+h_{2}+m\right) \\
c-\left(a+b+c+h_{2}+m\right) \\
a-\left(a+b+c+h_{3}+m\right) b-\left(a+b+c+h_{3}+m\right) \\
c-\left(a+b+c+h_{3}+m\right) .
\end{array}\right.
$$

Projizieren wir die so erhaltenen Punkte $\beta$ ) neuerdings wieder von allen einfachen Tripelpunkten des Systems $(a+h, b+h, c+h)$ aus, indem wir als Projektionszentrum irgend einen Tripelpunkt $a-k, b-k, c-k$ nehmen und in den erhaltenen Ausdrücken $k$ variabel sein lassen, so erhalten wir

$$
\begin{aligned}
& a-\left[-k-\left(h_{1}+m\right)\right] b-\left[-k-\left(h_{1}+m\right)\right] c-\left[-k-\left(h_{1}+m\right)\right] \\
& a-\left[-k-\left(h_{2}+m\right)\right] b-\left[-k-\left(h_{2}+m\right)\right] c-\left[-k-\left(h_{2}+m\right)\right] \\
& a-\left[-k-\left(h_{3}+m\right)\right] b-\left[-k-\left(h_{3}+m\right)\right] c-\left[-k-\left(h_{3}+m\right)\right]
\end{aligned}
$$

oder, wenn wir die Konstanten $h_{i}+m$ jedesmal durch einen Buchstaben $l_{i}$ ersetzen $-i=1,2,3$ -

$$
\gamma)\left\{\begin{array}{lll}
a+l_{1}+k & b+l_{1}+k & c+l_{1}+k \\
a+l_{2}+k & b+l_{2}+k & c+l_{2}+k \\
a+l_{3}+k & b+l_{3}+k & c+l_{3}+k
\end{array}\right.
$$

$\gamma)$ stellt bei variablem $k$ ein solches Tripelpunktsystem zweiten Grades dar; jedes $k$ ergibt drei Dreiecke, die zusammen einen Tripelpunkt zweiten Grades bilden.

Anmerkung. In ganz analoger Weise lassen sich Tripelpunkte 3., 4., ... $n^{\text {ten }}$ Grades bilden.

14. Sind nun z. B. zwei derartige Tripelpunkte zweiten Grades $k_{1}$ und $k_{2}$ gegeben:

$$
\begin{array}{llll}
\left.\delta_{1}\right) & a+l_{1}+k_{1} & b+l_{1}+k_{1} & c+l_{1}+k_{1} \\
\left.\delta_{2}\right) & a+l_{2}+k_{1} & b+l_{2}+k_{1} & c+l_{2}+k_{1} \\
\left.\delta_{3}\right) & a+l_{3}+k_{1} & b+l_{3}+k_{1} & c+l_{3}+k_{1} \\
\left.\delta_{1}^{\prime}\right) & a+l_{1}+k_{2} & b+l_{1}+k_{2} & c+l_{1}+k_{2} \\
\left.\delta_{2}^{\prime}\right) & a+l_{2}+k_{2} & b+l_{2}+k_{2} & c+l_{2}+k_{2} \\
\left.\delta_{3}^{\prime}\right) & a+l_{3}+k_{2} & b+l_{3}+k_{2} & c+l_{3}+k_{2},
\end{array}
$$

so bestimmen diese zwei Tripelpunkte eine Tripelgerade und wir erhalten den dritten dieser Geraden zugehörigen Tripelpunkt, 
wenn wir in der uns geläufigen Weise Dreieck $\delta_{1}$ mit Dreieck $\delta_{2}^{\prime}$ and mit Dreieck $\delta_{3}^{\prime}$ und ebenso $\delta_{2}$ mit $\delta_{3}^{\prime}$ verbinden.

$\delta_{1}$ und $\delta_{2}^{\prime}$, ebenso wie $\grave{\delta}_{2}$ and $\delta_{1}^{\prime}$, ergeben:

$$
\begin{aligned}
& a-\left[a+b+c+l_{1}+l_{2}+k_{1}+k_{2}\right], \\
& b-\left[a+b+c+l_{1}+l_{2}+k_{1}+k_{2}\right], \\
& c-\left[a+b+c+l_{1}+l_{2}+k_{1}+k_{2}\right]
\end{aligned}
$$

oder wenn wir $+l_{1}+l_{2}+l_{3}-\left(l_{1}+l_{2}+l_{3}\right)$ hinzufügen :

$$
\begin{aligned}
&\left.\varepsilon_{3}\right) a+l_{3}-\left[a+b+c+l_{1}+l_{2}+l_{3}+k_{1}+k_{2}\right], \\
& b+l_{3}-\left[a+b+c+l_{1}+l_{2}+l_{3}+k_{1}+k_{2}\right], \\
& c+l_{3}-\left[a+b+c+l_{1}+l_{2}+l_{3}+k_{1}+k_{2}\right] .
\end{aligned}
$$

$\delta_{1}$ und $\delta_{3}^{\prime}$, ebenso wie $\delta_{3}$ und $\delta_{1}^{\prime}$, ergeben geradeso:

$$
\left.\varepsilon_{2}\right) a+l_{2}-M, b+l_{2}-M, c+l_{2}-M,
$$

wenn wir $a+b+c+l_{1}+l_{2}+l_{3}+k_{1}+k_{2}$ der Kürze wegen mit $M$ bezeichnen.

$\delta_{2}$ und $\delta_{3}^{\prime}$, ebenso wie $\delta_{3}$ und $\delta_{2}^{\prime}$, ergeben:

$$
\left.\varepsilon_{1}\right) a+l_{1}-M, \quad b+l_{1}-M, \quad c+l_{1}-M .
$$

In eindeatiger Weise veranlassen also die Tripelpunkte zweiten Grades $\delta$ and $\delta^{\prime}$ den Tripelpunkt $\varepsilon$, der mit ihnen auf derselben Tripelgeraden liegt. Nehmen wir schlieblich $k_{1}=k_{2}=k$, dann geht $\delta^{\prime}$ in $\delta$ über und $\varepsilon$ ist der Tangentialpunkt von $\delta$; statt $k_{1}+k_{2}$ muß es dann in den Ausdrücken $\varepsilon 2 k$ heißen. Wir erhalten also zu dem Tripelpunkte zweiten Grades $\delta$ seinen Tangentialpunkt $\varepsilon$, wenn wir $\delta_{1}$ mit $\delta_{2}$ und $\delta_{3}$ und $\delta_{2}$ mit $\delta_{3}$ verbinden. Soll schließlich dieser Tangentialpunkt $\varepsilon$ mit $\delta$ zusammenfallen, $\delta$ also ein Wendepunkt sein, so maß $\varepsilon_{1}$, das Ergebnis der Verbindung von $\delta_{2}$ mit $\delta_{3}$, zusammenfallen mit $\delta_{1}$; also muß

$$
a+l_{1}-\left[a+b+c+l_{1}+l_{2}+l_{3}+2 k\right] \equiv a+l_{1}+k
$$

ebenso $b+l_{1}-\left[a+b+c+l_{1}+l_{2}+l_{3}+2 k\right] \equiv b+l_{1}+k$

und $c+l_{1}-\left[a+b+c+l_{1}+l_{2}+l_{3}+2 k\right] \equiv c+l_{1}+k$ sein.

Aus jeder dieser drei Gleichungen ergibt sich:

$$
k=-\frac{a+b+c+l_{1}+l_{2}+l_{3}}{3}+\frac{p \omega+p^{\prime} \omega^{\prime}}{3} .
$$


Setzen wir nun

$$
\begin{aligned}
& l_{1}-\frac{a+b+c+l_{1}+l_{2}+l_{3}}{3}=k_{1}, \\
& l_{2}-\frac{a+b+c+l_{1}+l_{2}+l_{3}}{3}=k_{2}, \\
& l_{3}-\frac{a+b+c+l_{1}+l_{2}+l_{3}}{3}=l_{3},
\end{aligned}
$$

so ergeben sich demnach für die Wendepunkte unseres Tripelpunktsystems zweiten Grades die Ausdrücke

$\left.\zeta_{1}\right) a+k_{1}+\frac{p \omega+p^{\prime} \omega^{\prime}}{3}, \quad b+k_{1}+\frac{p \omega+p^{\prime} \omega^{\prime}}{3}, c+k_{1}+\frac{p \omega+p^{\prime} \omega^{\prime}}{3} ;$

$\left.\zeta_{2}\right) a+k_{2}+\frac{p \omega+p^{\prime} \omega^{\prime}}{3}, \quad b+k_{2}+\frac{p \omega+p^{\prime} \omega^{\prime}}{3}, c+k_{2}+\frac{p \omega+p^{\prime} \omega^{\prime}}{3}$;

$\left.\zeta_{3}\right) a+k_{3}+\frac{p \omega+p^{\prime} \omega^{\prime}}{3}, \quad b+k_{3}+\frac{p \omega+p^{\prime} \omega^{\prime}}{3}, c+k_{3}+\frac{p \omega+p^{\prime} \omega^{\prime}}{3}$.

Diese Punkte decken sich aber mit den Punkten $g$ ) am Ende von $\$ 12$. Die neun Wendepunkte eines Tripelpunktsystems zweiten Grades bestehen also aus neun Tripelgeraden eines Systems ersten Grades, die in einem derartigen Zusammenhange untereinander stehen, dafo man aus einer die acht anderen erhält, wenn man die ersten von einem der neun einfachen Wendepunkte, z. B. dem Wendepunkte 0, aus projiziert; sodann diese Projektion neuerdings wieder von den acht anderen Wendepunkten aus projiziert [vgl. den Schluh von 12)].

Dieses Ergebnis steht mit unseren früheren Wahrnehmungen im Einklange: Die drei Schnittpunkte einer Geraden mit der $C_{3}$ bilden zusammen einen Tripelwendepunkt ersten Grades; es ist also folgerichtig, dab ebenso die drei Tripelpunkte einer einfachen Tripelgeraden einen Tripelwendepunkt zweiten Grades bilden.

15. Wir können nunmehr die in 12) erörterten Beziehungen zwischen den Tripelwendepunktsystemen ersten Grades $\varphi$ ) und ' $\varphi^{\prime}$ ) und dem zugehörigen Tripelwendepunktsystem zweiten Grades $g$.

$$
\text { 甲) }\left\{\begin{array}{l}
-a+\frac{a+b+c+p \omega+\underline{p^{\prime} \omega^{\prime}}}{3} \\
-b+\frac{a+b+c+p \omega+p^{\prime} \omega^{\prime}}{3} \\
-c+\frac{a+b+c+p \omega+p^{\prime} \omega^{\prime}}{3}
\end{array}\right.
$$




$$
\left\{\begin{array}{l}
-k_{1}-\frac{a+b+c+p \omega+p^{\prime} \omega^{\prime}}{3} \\
-k_{2}-\frac{a+b+c+p \omega+p^{\prime} \omega^{\prime}}{3} \\
-k_{3}-\frac{a+b+c+p \omega+p^{\prime} \omega^{\prime}}{3} \\
a+k_{2}+\frac{p \omega+p^{\prime} \omega^{\prime}}{3}, b+k_{2}+\frac{p \omega+p^{\prime} \omega^{\prime}}{3}, c+k_{2}+\frac{p \omega+p^{\prime} \omega^{\prime}}{3} \\
a+k_{1}+\frac{p \omega+p^{\prime} \omega^{\prime}}{3}, b+k_{1}+\frac{p \omega+p^{\prime} \omega^{\prime}}{3}, c+k_{1}+\frac{p \omega+p^{\prime} \omega^{\prime}}{3} \\
a+\frac{p \omega+p^{\prime} \omega^{\prime}}{3}, b+k_{3}+\frac{p \omega+p^{\prime} \omega^{\prime}}{3}, c+k_{3}+\frac{p \omega+p^{\prime} \omega^{\prime}}{3}
\end{array}\right.
$$

kurz so aussprechen:

Hat man zwei Tripelwendepunktsysteme 1. Grades $\varphi$ ) und $\varphi^{\prime}$ ) and verbindet jedend er 27 Puntedes einen Systems mit jedem der 27 Punktedes anderen, so bilden die dritten Schnittpunkte derVerbindungslinien ein Tripelwendepunktsystem zweiten Gradesg). Wir wollen dieses Resultat noch etwas genauer betrachten.

Die zwei Systeme $\varphi$ ) und $\varphi^{\prime}$ ) veranlassen, wenn wir jeden einfachen Punkt von $\varphi$ mit jedem von $\varphi^{\prime}$ verbinden, $27^{2}$ Verbindungslinien. Da nun jede dieser Geraden dureb einen der 81 Punkte des Tripelwendepunktsystems zweiten Grades $g$ geht, so gehen also allemal 9 dieser $27^{2}$ Verbindungslinien durch einen und denselben Punkt von g). Dalb das tatsächlich der Fall ist, kann man aus den Ausdrücken $\varphi, \varphi^{\prime}$ und $g$ ohne weiteres ablesen. So z. B. gehen durch den Punkt $a+k_{1}$ von $\left.g\right) 9$ Geraden nach den 9 Punkten von $\varphi):-a+\frac{a+b+c+p \omega+p^{\prime} \omega^{\prime}}{3}$, die sich ergeben, wenn man $p$ und $p^{\prime}$ jeden der drei Werte 1, 2, 3 gibt. $a+k_{1}$ und $-a+\frac{a+b+c+p \omega+p^{\prime} \omega^{\prime}}{3}$ ergeben aber für die dritten Schnittpunkte der. Verbindungslinien den Ausdruck von $\varphi^{\prime}$ ):

$$
-k_{1}-\frac{a+b+c+p \omega+p^{\prime} \omega^{\prime}}{3} \text {. }
$$

Die Frage, wie viele von diesen $27^{2}$ Linien reell und imaginür sind, ist leicht zu entscheiden, da wir in unserer Konfiguration die Tripelwendepunktsysteme ersten Grades $\varphi$ ) and $\varphi^{\prime}$ ) beliebig annehmen dürfen und ein beliebiger imaginärer Punkt, mit einem zweiten beliebigen imaginären Punkt verbunden, natürlich eine imaginäre Gerade liefert. Nun hat jedes der Tripelwendepunktsysteme $\varphi$ ) und $\varphi^{\prime}$ ) drei reelle und sechs imaginäre Geraden; die drei reellen Geraden haben entweder je drei reelle Punkte oder 
je einen; unter den $27^{2}$ Geraden sind also höchstens 81 reell; möglich ist auch, daß nur 27 oder auch daß nur 9 reell sind.

Daß tatsächlich $\varphi$ ) und $\varphi^{\prime}$ ) bei der Herstellung der Konfiguration $\left.\left.\left.[\varphi), \varphi^{\prime}\right), g\right)\right]$ beliebig angenommen werden dürfen, erkennen wir, wenn wir die Ausgangstripelgerade

$$
\begin{array}{lll}
a+k_{1} & b+k_{1} & c+k_{1} \\
a+k_{2} & b+k_{2} & c+k_{2} \\
a+k_{3} & b+k_{3} & c+k_{3}
\end{array}
$$

betrachten. Mit dieser Tripelgeraden ist auch das Parametersystem

$$
\text { ๑) }\left\{\begin{array}{l}
-a+\frac{a+b+c+p \omega+p^{\prime} \omega^{\prime}}{3} \\
-b+\frac{a+b+c+p \omega+p^{\prime} \omega^{\prime}}{3} \\
-c+\frac{a+b+c+p \omega+p^{\prime} \omega^{\prime}}{3}
\end{array}\right.
$$

bestimmt. Dieses System ist aber, wie man sieht, schon mit dem Tripelpunkt $a b c$ gegeben, gehört also zu sämtlichen Tripelgeraden des Tripelpunktsystems $(a, b, c)$. Selbstverständlich erhalten wir aber im allgemeinen zu $\varphi$ ) und je zwei Tripelpunktsystemen zweiten Grades $g_{1}$ ) and $g_{2}$ ) zwei verschiedene Systeme $\varphi^{\prime}$. Wir werden also $z$ a dem System $\varphi$ ) so viele Systeme $\varphi^{\prime}$ ) konstruieren können, als in dem Tripelpunktsystem $(a, b, c)$ Tripelwendepunktsysteme zweiten Grades g) sich bilden lassen. Man wird also tatsächlich zur Bildung der Konfiguration $\left.\left.\left.[\varphi), \varphi^{\prime}\right), g\right)\right] \varphi$ ) und $\varphi^{\prime}$ ) beliebig annehmen dürfen. Wir erkennen gleichzeitig, weil $\varphi$ ) durch $\left.a, b, c, \varphi^{\prime}\right)$ durch $k_{1}$, $k_{2}, k_{3}$ bestimmt ist:

Es gibt neun Tripelgeraden des Tripelpunktsystems $(a, b, c)$, deren konjugierte Tripelgeraden wiederum demselben Tripelpunktsystem $\left(k_{1}, k_{2}, k_{3}\right)$ angehören. Es sind dies die Geraden

$$
\begin{aligned}
& a+k_{1}+\frac{p \omega+p^{\prime} \omega^{\prime}}{3}, b+k_{1}+\frac{p \omega+p^{\prime} \omega^{\prime}}{3}, c+k_{1}+\frac{p \omega+p^{\prime} \omega^{\prime}}{3} \\
& a+k_{2}+\frac{p \omega+p^{\prime} \omega^{\prime}}{3}, b+k_{2}+\frac{p \omega+p^{\prime} \omega^{\prime}}{3}, c+k_{2}+\frac{p \omega+p^{\prime} \omega^{\prime}}{3} \\
& a+k_{3}+\frac{p \omega+p^{\prime} \omega^{\prime}}{3}, b+k_{3}+\frac{p \omega+p^{\prime} \omega^{\prime}}{3}, c+k_{3}+\frac{p \omega+p^{\prime} \omega^{\prime}}{3},
\end{aligned}
$$

deren jede sich durch einen bestimmten Wert von $p$ und $p^{\prime}$ ergibt, and die zusammen das Tripelwendepunktsystem zweiten Grades $g$ ) bilden. 
Die erste dieser Tripelgeraden ist bestimmt durch die drei Tripelpunkte

$$
\text { 1) } \begin{array}{lll}
a+k_{1} & b+k_{1} & c+k_{1} \\
a+k_{3} & b+k_{2} & c+k_{2} \\
a+k_{3} & b+k_{3} & c+k_{3},
\end{array}
$$

wo jede wagrechte Reihe einen Tripelpankt der Geraden gibt und $k_{3}=-\left(a+b+c+k_{1}+k_{2}\right)$ ist. Die zu 1) gehörige konjugierte Gerade setzt sich aus den Tripelpunkten zusammen:

$$
\text { 1) } \begin{array}{lll}
k_{1}+a & k_{2}+a & k_{3}+a \\
k_{1}+b & k_{2}+b & k_{3}+b \\
k_{1}+c & k_{2}+c & k_{3}+c,
\end{array}
$$

die dem Tripelpunktsystem $\left(k_{1}, k_{2}, k_{3}\right)$ angehören.

Eine zweite der neun erwähnten Geraden des Tripelpunktsystems $(a, b, c)$ ist gegeben durch:

$$
\text { 2) } \begin{array}{lll}
a+\left(k_{1}+\frac{\omega}{3}\right) & b+\left(k_{1}+\frac{\omega}{3}\right) & c+\left(k_{1}+\frac{\omega}{3}\right) \\
a+\left(k_{2}+\frac{\omega}{3}\right) & b+\left(k_{2}+\frac{\omega}{3}\right) & c+\left(k_{2}+\frac{\omega}{3}\right) \\
a+\left(k_{3}+\frac{\omega}{3}\right) & b+\left(k_{3}+\frac{\omega}{3}\right) & c+\left(k_{3}+\frac{\omega}{3}\right)
\end{array}
$$

wo die eingeklammerten Ausdrücke die Parameter der Tripelpunkte bedeuten, während $a, b, c$ der Ausgangstripelpunkt ist. Die zu 2) konjugierte Tripelgerade hat die drei Tripelpunkte:

$$
\begin{array}{rlr}
2^{\prime} k_{1}+\left(a+\frac{\omega}{3}\right) & k_{2}+\left(a+\frac{\omega}{3}\right) & k_{3}+\left(a+\frac{\omega}{3}\right) \\
k_{1}+\left(b+\frac{\omega}{3}\right) & k_{2}+\left(b+\frac{\omega}{3}\right) & k_{3}+\left(b+\frac{\omega}{3}\right) \\
k_{1}+\left(c+\frac{\omega}{3}\right) & k_{2}+\left(c+\frac{\omega}{3}\right) & k_{3}+\left(c+\frac{\omega}{3}\right)
\end{array}
$$

wo wieder die eingeklammerten Ausdrücke die Parameter, $k_{1}, k_{2}, k_{3}$ der Ausgangspunkt ist. Die zu 2) konjugierte Tripelgerade 2') gehört also wieder dem Tripelpunktsystem $\left(k_{1}, k_{2}, k_{3}\right)$ an u. s. w.

16. Bevor ich zu einer anderen Gattung von Punkts stemen übergehe, will ich an einem Beispiel zeigen, wie sich unsere Tripelpunkttheorie als Hilfsmittel zur Ableitung von Sätzen der gewöhnlichen Punkttheorie verwerten läßt. Sind $t_{1}, t_{2}, t_{3}, t_{4}$ die vier von einem Punkte $a^{\prime}$ der $C_{3}$ ausgehenden weiteren vier Tangenten, mit den Berührungspunkten $a_{1}, a_{2}, a_{3}, a_{4}$, so gibt es einen Kegel- 
schnitt durch $a_{1}, a_{2}, a_{3}, a_{4}$, der in $a^{\prime}$ berührt. Hat ebenso eine Gruppe von vier Tripeliangenten die Berührungstripelpunkte:
1) $a, b, c$;
2) $a+\frac{\omega}{2}$
$b+\frac{w}{2}, c+\frac{\omega}{2}$
3) $a+\frac{\omega^{\prime}}{2}$,
$b+\frac{\omega^{\prime}}{2}$
$c+\frac{\omega^{\prime}}{2}$
4) $a+\frac{\omega+\omega^{\prime}}{2}$,
$b+\frac{\omega+\omega^{\prime}}{2}$,
$c+\frac{\omega+}{2} \omega^{\prime}$

und den gemeinsamen Tangentialtripelpunkt 5) $a-(a+b+c)$, $b-(a+b+c), c-a+b+c-$ der Einfachheit wegen wurde der Ausgangstripelpunkt $a, b, c$, der den Parameter 0 hat, verwendet - , so läßßt sich eine $C_{6}$ durch die vier Tripelpunkte 1) bis 4) legen, die die $C_{3}$ in den Eckpunkten des Dreiecks 5) berührt. Das läfot sich am einfachsten durch den Nachweis zeigen, dab für die Parameter unserer sechs Tripelpunkte, der gemeinsame Tangentialtrjpelpunkt 5) zweifach gezählt, die Gleichung besteht $k_{1}+k_{2}+\cdots+k_{6} \equiv-2(a+b+c)\left(\bmod \omega, \omega^{\prime}\right)(\mathrm{s} . \S 8$, Gleichung 2$)$.

Diese Summe der Parameter ist in unserem Falle:

$$
0+\frac{\omega}{2}+\frac{\omega^{\prime}}{2}+\frac{\omega+\omega^{\prime}}{2}+2 \cdot[-(a+b+c)]=-2(a+b+c) \text {. }
$$

Nehmen wir nun als Ausgangsdreieck ein Dreieck $a b c$ mit zwei unendlich benachbarten Eckpunkten $b$ und $c$, so findet dieses $\mathrm{Zu}$ sammenfallen der zwei $b$ und $c$ entsprechenden Eckpunkte natürlich bei sämtlichen Tripelpunkten des Systems $(a, b, c)$ statt; es gibt also (s. Fig. 2) eine $C_{6}$, die durch die Tripelpunkte $a_{1} c_{1} c_{1}, a_{2} c_{2} c_{2}, a_{3} c_{3} c_{3}, a_{4} c_{4} c_{4}$ je einfach und durch den gemeinsamen Tangentialtripelpunkt $a^{\prime} c^{\prime} c^{\prime}$ doppelt

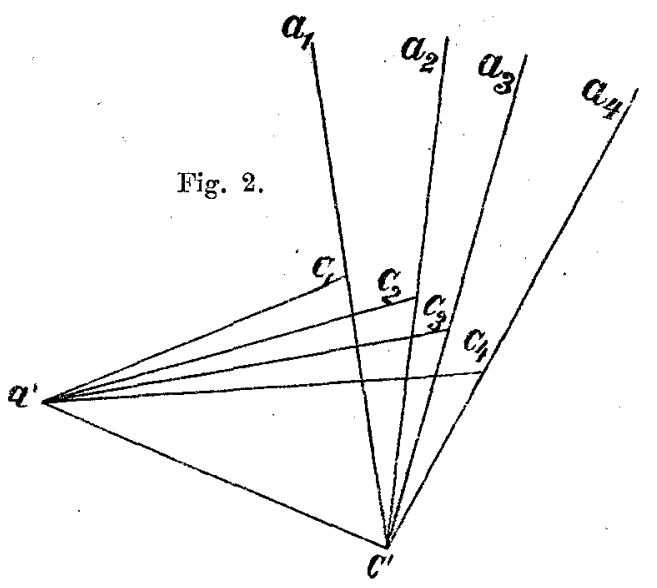
hindurchgeht, die also mit der $C_{3}$ die Punkte $a_{1}, a_{2}, a_{3}, a_{4}$ einfach, die Punkte $c_{1}, c_{2}, c_{3}, c_{4}, a^{\prime}$ zweifach und den Punkt $c^{\prime}$ vierfach gemeinsam hat; dabei ist $a^{\prime}$ der Tangentialpunkt der vier Punkte $c_{1}, c_{2}, c_{3}, c_{4}$.

Nehmen wir nun eine zerfallene $C_{6}$, von welcher die Tangenten $\left(a^{\prime}, c_{1}\right)$ und $\left(a^{\prime}, c_{2}\right)$ ferner die Geraden $\left(c^{\prime}, a_{3}\right)$ und $\left(c^{\prime}, a_{4}\right)$ Bestand- 
teile sind, so bleibt ein Kegelschnitt.übrig, der durch $a_{1}, a_{2}, c_{3}, c_{4}$ und zweimal durch $c^{\prime}$ geht; also:

Hat man ein Tangentenquadrupel $t_{1}, t_{2}, t_{3}, t_{4}$ mit gemeinsamem Tangentialpunkt $a^{\prime}$ (s. Fig. 2) und bezw. den Berührungspunkten $c_{1}, c_{2}, c_{3}, c_{4}$, und legt mandurch diese vier Berührungspunkte irgend ein Quadrupel von Geraden $e^{\prime} c_{1} a_{1}, c^{\prime} c_{2} a_{2}, c^{\prime} c_{3} a_{3}, c^{\prime} c_{4} a_{4}$, beliebig, weil $c^{\prime}$ irgend ein Punktder $C_{3}$ ist, während $a_{1}, a_{2} \ldots$ diedritten Schnittpunkte der Geraden $c^{\prime} c_{1}, c^{\prime} c_{2} \ldots$ sind, so existieren die sechs Kegelschnitte:

$$
\begin{aligned}
& c^{\prime} c^{\prime} c_{1} c_{2} a_{3} a_{4} \\
& c^{\prime} c^{\prime} c_{1} c_{3} a_{2} a_{4} \\
& c^{\prime} c^{\prime} c_{1} c_{4} a_{2} a_{3} \\
& c^{\prime} c^{\prime} c_{2} c_{3} a_{1} a_{4} \\
& c^{\prime} c^{\prime} c_{2} c_{4} a_{1} a_{3} \\
& c^{\prime} c^{\prime} c_{3} c_{4} a_{1} a_{2} .
\end{aligned}
$$

Anmerkung: Lassen wir $c^{\prime}$ mit dem Tangentialpunkt $a^{\prime}$ znsammenfallen, so fallen die Punktgruppen $a$ und $c$ zusammen und statt der sechs Kegelschnitte erhalten wir als speziellen Fall den einen bekannten Kegelschnitt $\left(c_{1}, c_{2}, c_{3}, c_{4}, a^{\prime}, a^{\prime}\right)$.

17. Fortsetzung. Er-

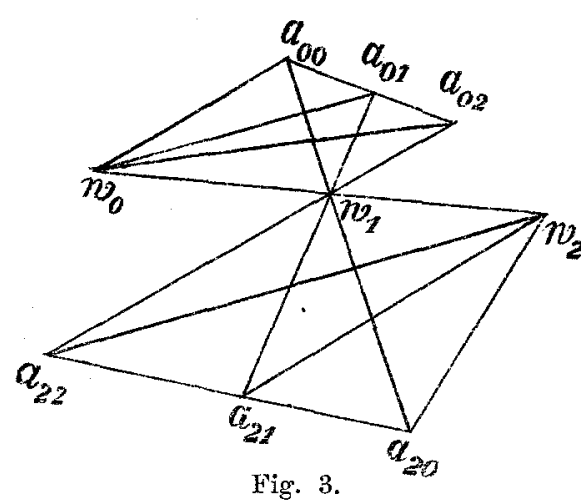
wähnenswert sind die Beziehungen, die sich für die Lage dreier in einer Geraden liegenden Wendepunkte und der Punkte ihrer harmonischen Polaren aus diesem Satze ergeben (s. die schematische Fig. 3).

Seien die Wendepunkte $w_{0}, w_{1}, w_{2}$ und die Punkte der zu $w_{0}$ und $w_{2}$ gehörigen harmonischen Polaren bezw. $a_{00}, a_{01}, a_{02}$ und $a_{20}, a_{21}, a_{22}$, so daß die Punkte $a_{00}, w_{1}, a_{20}$; $a_{01}, w_{1}, a_{21} ; a_{02}, w_{1}, a_{22}$ jedesmal in einer Geraden liegen. Wählen wir alsdann als das Tangentenquadrupel die Wendetangente und die weiteren Tangenten in $w_{0}$, die also die Berührungspunkte $w_{0}, a_{00}, a_{01}, a_{02}$ haben, und wählen als Punkt $c^{\prime}$ (s. Fig. 2) den Wendepunkt $w_{1}$, so geht durch jedes der folgenden sechs Punktquadrupel ein Kegelschnitt, der die $C_{3}$ in $w_{1}$ berührt:

$$
\begin{array}{ll}
w_{0} a_{00} a_{21} a_{22} & w_{2} a_{20} a_{01} a_{02} \\
w_{0} a_{01} a_{20} a_{22} & w_{2} a_{21} a_{00} a_{02} \\
w_{0} a_{02} a_{21} a_{20} & w_{2} a_{22} a_{01} a_{00} .
\end{array}
$$


Wie leicht ersichtlich ist, erhält man dieselben sechs Kegelschnitte, wenn man das von $v_{0}$ ausgehende Tangentenquadrupel durch das von $w_{2}$ ausgehende ersetzt.

Eine Inflexionsachse mit den zugehörigen drei harmonischen Polaren gibt also Anlaß zu $18 \mathrm{Kegel-}$ schnitten, von denen je sechs in einem der drei auf der Inflexionsachse liegenden Wendepunkte berühren und deren übrige vier Schnittpunkte Punkte der harmonischen Polaren sind. Da dureh jeden Wendepunkt vier Infexionsachsengehen, so berühren demnach in jedem Wendepunkte 24 solehe Kegelschnitte. IhreZahl beträgt also im ganzen 216. Davon. sind aber höchstens die $18 \mathrm{zu}$ der reellen Inflexionsachse gehörigen reell.

16 a). Eine weitere bemerkenswerte Gruppe von vier Kegelschnitten erhalten wir, wenn wir den allgemeinen Satz, der die Grundlage von 16) bildet, in anderer Weise spezialisieren.

Dieser allgemeine Satz besagt, daß die Gruppe der vier Punkte (s. Fig. 2) $a, a+\frac{\omega}{2}, a+\frac{\omega^{\prime}}{2}, a+\frac{\omega+\omega^{\prime}}{2}$ je einfach zählend, das Quadrupel der Punkte $c, c+\frac{\omega}{2}, c+\frac{\omega^{\prime}}{2}, c+\frac{\omega+\omega^{\prime}}{2}$ je zweifach gerechnet, der Punkt $a^{\prime}$ zweifach und der Punkt $c^{\prime}$ vierfach zählend 18 assoziierte Punkte bilden. So oft nun von diesen 18 Punkten viermal drei Punkte, z. B. die Punkte $a^{\prime}, c, c ; c^{\prime}, c+\frac{\omega}{2}, a+\frac{\omega}{2}$; $c^{\prime}, c+\frac{\omega^{\prime}}{2}, a+\frac{\omega^{\prime}}{2} ; c^{\prime}, c+\frac{\omega+\omega^{\prime}}{2}, a+\frac{\omega+\omega^{\prime}}{2}$, auf je einer Geraden liegen, so oft liegen die übrigen sechs Punkte auf einer $C_{2}$; in unserem Beispiele heiben diese sechs Punkte $a^{\prime}, c^{\prime}, a, c+\frac{\omega}{2}$, $c+\frac{\omega^{\prime}}{2}, c+\frac{\omega+\omega^{\prime}}{2}$. Also:

Hat man ein Quadrupel von vier von einem Punkte $\alpha^{\prime}$ der $C_{3}$ ausgehenden Tangenten, mit den Berührungspunkten $c_{1}, c_{2}, c_{3}, c_{4}$, und projiziertman diese vier Punkte von irgend einem Punkte $e^{\prime}$ der Kurve auf dieselbe, so erhält man das Punktquadrupel $a_{1}, a_{2}, a_{3}, a_{4}$. Es gehen dann dureh $a^{\prime}$ und $c^{\prime}$ vier Kegelschnitte, die folgende weitere Gruppen von vier Punkten ausscheiden:

$$
\begin{aligned}
& a_{1} c_{2} c_{3} c_{4} \\
& a_{2} c_{1} c_{3} c_{4} \\
& a_{3} c_{1} c_{2} c_{4} \\
& a_{4} c_{1} c_{2} c_{3} .
\end{aligned}
$$


17. $\mathrm{Zu}$ weiteren Konfigurationen führt der allgemeine Satz in 16), wenn wir in anderer Weise spezialisieren.

Ist $a^{\prime} b^{\prime} c^{\prime}$ der Tangentialtripelpunkt zu $a_{1} b_{1} c_{1}, a_{2} b_{2} c_{2}, a_{3} b_{3} c_{3}$, $a_{4} b_{4} c_{4}$, dann gehen also durch $a^{\prime}$ die vier Geraden $b_{1} c_{1}, b_{2} c_{2}, b_{3} c_{3}, b_{4} c_{4}$, durch $b^{\prime} a_{1} c_{1} \ldots$ u. s. w. und $a_{1}, b_{1}, c_{1} ; a_{2}, b_{2}, c_{2} ; a_{3}, b_{3}, c_{3} ; a_{4}, b_{4}, c_{4}$; $a^{\prime}, b^{\prime}, c^{\prime} ; a^{\prime}, b^{\prime}, c^{\prime}$ sind 18 assozilerte Punkte. Nun liegen z. $\mathrm{B}$. $c^{\prime}, a_{3}, b_{3} ; c^{\prime}, a_{4}, b_{4} ; b^{\prime}, a_{1}, c_{1} ; b^{\prime}, a_{2}, c_{2}$ auf je einer Geraden; also liegen die übrigen sechs Punkte auf einem Kegelschnitt; es sind dies die Punkte $a^{\prime}, a^{\prime} c_{3}, c_{4}, b_{1}, b_{2}$. Solcher Kegelschnitte, die in $a^{\prime}$ berühren, gibt es sechs; jeder geht noch durch eine der folgenden Gruppen von vier weiteren Punkten:

$$
\begin{aligned}
& b_{1} b_{2} c_{3} c_{4} \\
& b_{1} b_{3} c_{2} c_{4} \\
& b_{1} b_{4} c_{2} c_{3} \\
& b_{2} b_{3} c_{1} c_{4} \\
& b_{2} b_{4} c_{1} c_{3} \\
& b_{3} b_{4} c_{1} c_{2} .
\end{aligned}
$$

In $b^{\prime}$ und $c^{\prime}$ berühren ebenfalls je sechs ähnlich zu bestimmende Kegelschnitte.

$17 a$ ). Splittern wir aber von einer durch die 18 assoziierten Punkte zu legenden $C_{6}$ die Geraden $c^{\prime} a_{3} b_{3}, c^{\prime} a_{4} b_{4}, a^{\prime} b_{1} c_{1}, b^{\prime} a_{2} c_{2}$ $\mathrm{ab}$, so bleibt ein Kegelschnitt übrig, der durch $a^{\prime}, b^{\prime}, c_{3}, c_{4}, a_{1}, b_{2}$ geht. Solcher Kegelschnitte durch $a^{\prime}$ und $b^{\prime}$ gibt es zwölf; die zugehörigen weiteren Schnittpunktquadrupel heißen:

$$
\begin{aligned}
& c_{1} c_{2} a_{3} b_{4} \\
& c_{1} c_{2} a_{4} b_{3} \\
& c_{1} c_{3} a_{2} b_{4} \\
& c_{1} c_{3} a_{4} b_{2} \\
& c_{1} c_{4} a_{2} b_{3} \\
& c_{1} c_{4} a_{3} b_{2} \\
& c_{2} c_{3} a_{1} b_{4} \\
& c_{2} c_{3} a_{4} b_{1} \\
& c_{2} c_{4} a_{1} b_{3} \\
& c_{2} c_{4} a_{3} b_{1} \\
& c_{3} c_{4} a_{1} b_{2} \\
& c_{3} c_{4} a_{2} b_{1} .
\end{aligned}
$$

Ebenso viele ähnlich zu bestimmende Kegelschnitte gehen durch die zwei Punkte $a^{\prime}$ und $c^{\prime}$ und durch die zwei Punkte $b^{\prime}$ und $c^{\prime}$.

Wenn wir der Kürze wegen die fünf Dreiecke $a_{1} b_{1} c_{1} \ldots a_{4} b_{4} c_{4}$, $a^{\prime} b^{\prime} c^{\prime}$ mit $d_{1}, d_{2}, d_{3}, d_{4}, d^{\prime}$ bezeichnen, so können wir zusammenfassend folgendes feststellen: 
Durch je sechs Eckpunkte von fünf der $C_{3}$ einbeschriebenen Dreiecken $d_{1}, d_{2}, d_{3}, d_{4}, d^{\prime}$, von denen die vier ersten dem letzten umbeschrieben sind, geben im ganzen $54 \mathrm{Kegelschnitte.} \mathrm{Sie} \mathrm{teilen} \mathrm{sich} \mathrm{in} \mathrm{eine}$ Gruppe $A$ von dreimal sechs Kegelschnitten, die je in einem Eckpunkte von $d^{\prime}$ berühren (welcher also dann für zwei Eckpunktezählt) und in eineGruppe $B$ von 36 Kegelschnitten, von welchen allemal zwölf durchein- und dasselbe Eckpunktpaar von $d^{\prime}$ hindurchgehen.

18. Es erhebt sich nun die Frage: Ist das Dreieck die einzige Figur, die als Grundlage für derartige Theorien benützt werden kann, oder gibtes nicht Systeme von der $C_{3}$ einbeschriebenen Vielecken jeder Art, bei denen solche Analogien mit der Theorie der einfachen Punkte der $C_{3}$ möglich sind? Wir gehen zur Entscheidung dieser Frage ron irgend einem System der $C_{3}$ einbeschriebener $n$-Ecke aus:

$$
\text { 1) } a_{1}+h, a_{2}+h, a_{3}+h, \ldots a_{p}+h \ldots a_{n}+h
$$

wobei $a_{1} a_{2} a_{3} \ldots a_{n}$ das Ausgangsvieleck, $h$ der variable Parameter sei.

Halten wir nun für das aufzufindende neue System an der Definition fest, daß zu einem Vieleck das Tangentialvieleck durch Bestimmung der dritten Schnittpunkte der Seiten erhalten wird, so mub dasselbe System auch gegeben sein durch

2) $-2 h-\left(a_{1}+a_{2}\right),-2 h-\left(a_{2}+a_{3}\right),-2 h--\left(a_{3}+a_{4}\right), \ldots$;

dabei ist $-2 h$ der variable Parameter. Die Systeme 1) und 2) werden nun identisch, wenn:

$$
\begin{aligned}
& a_{1}+a_{2}=-a_{1} \\
& a_{2}+a_{3}=-a_{2} \text { u.s.w.; }
\end{aligned}
$$

wir erhalten alsdann:

$$
\begin{aligned}
& a_{2}=-2 a_{1}, \\
& a_{3}=-2 a_{2}=4 a_{1} \quad \text { u.s.w.; }
\end{aligned}
$$

d. h. das $n$-Ecksystem, zu dem wir so kommen, ist von der Art, dafo das Ausgangs- $n$-Eck ein der $C_{3}$ um- und einbeschriebenes Vieleck ist. Ein solches $n$-Eck hat also die Eckpunkte:

$$
\begin{aligned}
& a_{1}=a \cdot 2^{0}(-1)^{0} \quad a_{p}=a \cdot 2^{p-1}(-1)^{p-1} \\
& a_{2}=a \cdot 2^{1}(-1)^{1} \quad:::::::::: \vdots \\
& a_{3}=a \cdot 2^{2}(-1)^{2} \quad a_{n}=a \cdot 2^{n-1} \cdot(-1)^{n-1}
\end{aligned}
$$

der $(n+1)^{\text {te }}$ Eckpunkt heißt $a \cdot 2^{12} \cdot(-1)^{n}$; da dieser $(n+1)^{\text {te }}$ Eckpunkt mit dem ersten zusammenfallen muf, so haben wir 
$a \cdot 2^{n} \cdot(-1)^{n}=a+p \omega+p^{\prime} \omega^{\prime} ;$

$a\left[2^{n} \cdot(-1)^{n}-1\right]=p \omega+p^{\prime} \omega^{\prime}$;

$a=\frac{p \omega+p^{\prime} \omega^{\prime}}{2^{n} \cdot(-1)^{n}-1}$, wobei $p$ u. $p^{\prime}$ irgendwelche ganze Zahlen bedeuten.

19. Wir wollen der Kürze wegen die Bezeichnung a für $\frac{p \omega+p^{\prime} \omega^{\prime}}{2^{n} \cdot(-1)^{n}-1}$ beibehalten. Von. der Brauchbarkeit eines solchen $n$-Eckes nun zur Bildung eines polygonalen Punktsystems

$$
(a+h,-2 a+h, 4 a+h \ldots),
$$

in welchem das Polygon den Punkt vorstellt, kann man sich leicht überzeugen. Daß der Tangentialpunkt unseres polygonalen Punktes wieder dem System angehört, haben wir schon gesehen. Nehmen wir nun etwa zwei von einander getrennt liegende Polygone, $a+h_{1},-2 a+h_{1} \ldots ; a+h_{2},-2 a+h_{2} \ldots$, oder kurz: Das Polygon $h_{1}$ und das Polygon $h_{2}$, so läßt sich mittels dieser zweier Vielecke ein Vieleck $h_{3}$ konstruieren, das unserer Mannigfaltigkeit $(a+h,-2 a+h \ldots)$ angehört. Die Möglichkeiten, diese Konstruktionen $\mathrm{zu}$ bewerkstelligen, sind, wie wir sehen werden, verschieden, aber das Resultat ist immer dasselbe Vieleck $h_{3}$. $h_{1}, h_{2}, h_{3}$ bilden dann eine "polygonale Gerade", die durch die zwei Punkte $h_{1}$ und $h_{2}$ bestimmt ist. Ich will nun $h_{3}$ aus $h_{1}$ und $h_{2}$ konstruieren. Ich nehme von $h_{1}$ den Punkt $(-1)^{p} \cdot 2^{p} \cdot a+h_{1}$, also den $(p-1)^{\text {ten }}$ Punkt, von $(-1)^{a} 2^{0} . a-\frac{1}{1} h_{1}$ angefangen, von $h_{\mathrm{g}}$ den Punkt von der gleichen Ordnungszahl $(-1)^{p} 2^{p} \cdot a+h_{2}$, so ist der dritte Schnittpunkt der Verbindungslinie dieser zwei Punkte gegeben durch

$$
-h_{1}-h_{2}-2 \cdot(-1)^{p} \cdot 2^{p} \cdot a=-h_{1}-h_{2}+(-1)^{p+1} 2^{p+1} \cdot a
$$

aber dieser Punkt ist offenbar der $(p+2)^{\text {te }}$ Punkt des Vielecks - $\left(h_{1}+h_{2}\right)$, also des Vielecks $a-\left(h_{1}+h_{2}\right),-2 a-\left(h_{1}+h_{2}\right)$, $+4 a-\left(h_{1}+h_{2}\right) \ldots$

Wenn wir für $p$ alle Zahlen von 1 bis $n$ nehmen, so bekommen wir in dieser Weise alle Eckpunkte von $h_{3}$. Wir erkennen gleichzeitig die Bedingung, die für die Parameter $h_{1}, h_{2}, h_{3}$ bestehen muß, damit sie drei Punkte einer polygonalen Geraden darstellen:

$$
h_{1}+h_{2} \equiv-h_{3}\left(\bmod \omega, \omega^{\prime}\right) \text {; }
$$

das ist dieselbe Gleichung, die auch ausdrückt, daß die einfachen Punkte $h_{1}, h_{2}, h_{3}$ in einer Geraden liegen; die Analogie erstreckt sich hier also auch auf die Form der Gleichung. Gleichung

20. Daran knüpft sich naturgemüßb die Frage, was hat die 3) $h_{1}+h_{2}+\cdots+h_{3 m} \equiv 0\left(\bmod \omega, \omega^{\prime}\right)$, 
die beim einfachen Punktsystem ausdrückt, daf die Punkte mit den $3 m$ Parametern auf einer $C_{m}$ liegen, für unser polygonales System zu bedeuten? Was hat man unter einer polygonalen $C_{m}$ zu verstehen? Die Frage erledigt sich durch eine einfache Rechnung.

Nehmen wir $3 m$ Vielecke der Mannigfaltigkeit, mit den Parametern $h_{1}, h_{2}, h_{3} \ldots h_{3 m}$, so ist die Summe der Parameter ihrer Eckpunkte:

$$
\text { 4) } \begin{aligned}
3 m \cdot & {\left[a-2 a+4 a-\cdots+(-2)^{n-1} \cdot a\right]+} \\
& +n \cdot\left(h_{1}+h_{2}+h_{3}+\cdots+h_{3 m}\right) .
\end{aligned}
$$

Nun ist der erste Summand

$$
\begin{gathered}
3 m a\left[1-2+4 \cdots(-2)^{n-1}\right]= \\
=3 m a \frac{(-2)^{n}-1}{-3}=-m \cdot a\left[(-2)^{n}-1\right]= \\
=-m \frac{p \omega+p^{\prime} \omega^{\prime}}{(-2)^{n}-1}\left[\left(-2^{n}-1\right]=-m \cdot\left(p \omega+p^{\prime} \omega^{\prime}\right) \equiv 0\left(\bmod \omega, \omega^{\prime}\right) .\right.
\end{gathered}
$$

Die Summe 4) der $3 m n$ Parameter ist also $\equiv 0$, wenn

$$
\text { 3) } h_{1}+h_{2}+\cdots+h_{3 m} \equiv 0\left(\bmod \omega, \omega^{\prime}\right) \text { ist. }
$$

Also: Die Gleichung 3) drückt aus, daß die Eckpunkte der zu den $3 m$ Parametern gehörigen polygonalen Punkte die Schnittpunkte einer $C_{m n}$ mit der $C_{3}$ sind.

21. Bemerkenswert ist nun aber, daß zu zwei Vielecken $h_{1}$ und $h_{2}$ $h_{3}$ auf dreierlei Art gewonnen werden kann.

Wir betrachten wieder die drei Vielecke $h_{1}, h_{2}, h_{3}$, von der Art, daß $h_{3} \equiv-h_{1}-h_{2}\left(\bmod \omega, \omega^{\prime}\right)$

$$
h_{1} \text { habe die Eckpunkte: }
$$

$$
a_{1}^{\prime}=a+h_{1}, a_{2}^{\prime}=-2 a+h_{1}, \cdots a_{p+1}^{\prime}=(-2)^{p} \cdot a+h_{1}
$$

$h_{2}$ habe die Eckpunkte:

$$
a_{1}^{\prime \prime}=a+h_{2}, a_{2}^{\prime \prime}=-2 a+h_{2}, \cdots a_{p+1}^{\prime \prime}=(-2)^{p} \cdot a+h_{2}
$$

$h_{3}$ habe die Eckpunkte:

$$
a_{1}^{\prime \prime \prime}=a+h_{3}, a_{2}^{\prime \prime \prime}=-2 a+h_{3}, \cdots a_{p+1}^{\prime \prime \prime}=(-2)^{p} \cdot a+h_{3} ;
$$

$a$ ist, wie wir gesehen haben, $=\frac{p \omega+p^{\prime} \omega^{\prime}}{(-2)^{n}-1}$.

Dann ist:

$$
\begin{gathered}
-\left[(-2)^{p} a+h_{1}+(-2)^{p-1} \cdot a+h_{2}\right] \\
=-a\left[(-2)^{p-1} \cdot(-2+1)\right]-\left(h_{1}+h_{2}\right)=a(-2)^{p-1}+h_{3},
\end{gathered}
$$


d. h. der dritte Schnittpunkt der Verbindungslinie von $a_{p+1}$ und $a_{p}^{\prime \prime}$ ist $a_{p}^{\prime \prime \prime}$. Ein Eckpunkt $a_{p}^{\prime \prime \prime}$ des Vielecks $h_{3}$ wird also durch folgende Verbindungslinien von Eckpunkten der Vielecke $h_{1}$ und $h_{2}$ ausgeschnitten: 1) durch $a_{p-1}^{\prime} a_{p-1}^{\prime \prime}$; 2) durch $a_{p+1}^{\prime} a_{p}^{\prime \prime}$; 3) durch $a_{p}^{\prime} a_{p+1}^{\prime \prime}$. Die Zahl dieser Geraden ermäfigt sich auf 2, wenn $h_{1}$ und $h_{2}$ unendlich benachbart sind, weil alsdann $a_{p+1}^{\prime} a_{p}^{\prime \prime}$ und $a_{p}^{\prime} \quad a_{p+1}^{\prime \prime}$ zusammenfallen. Nehmen wir also wieder ein Vieleck $h$ und bezeichnen diesmal die Eckpunkte mit $b_{1} b_{2} \ldots b_{p-1} b_{p}$ $b_{p+1} \ldots b_{n}$; bezeichnen wir ferner die Eckpunkte des zugehörigen Tangentialvielecks $h^{\prime}$ mit $a_{1}^{\prime} a_{2}^{\prime} \ldots a_{p}^{\prime} \ldots a_{n}^{\prime} ;$ dann führen zu dem Punkte $a_{p}^{\prime}$ folgende zwei Geraden: 1) die Tangente in $b_{p-1}$;2) die Gerade $b_{p} \cdot b_{p+1}$.

22) Was die Wendepunkte des Systems betrifft, so sind dieselben natürlich gegeben durch $h=\frac{p \omega+p^{\prime} \omega^{\prime}}{3}$. Man erbält sie also in ihrer Gesamtheit, wenn man das um- und einbeschriebene Vieleck, welches das Ausgangspolygon bildet, ron einem Wendepunkt aus projiziert und die Projektion neverdings von allen Wendepunkten aus projiziert. Selbstverständlich lassen sich sämtliche Wendepunktbeziehungen auf unsere polygonalen Wendepunkte übertragen: je zwei derselben liegen auf einer polygonalen Inflexionsachse, die noch einen dritten enthält; es gibt zwölf solche Inflexionsachsen, durch jeden polygonalen Wendepunkt gehen vier u. s. w.

Folgender Satz ist aber auch ohne unsere Analogiebetrachtungen von Interesse: Zu jedem der $C_{3}$ um- und einbeschriebenen Vieleck $A^{\prime}$ stehen drei der Kurve einbeschriebene Vielecke $B_{1}$, $B_{2}, B_{3}$ von derselben Seitenzahl in der Beziehung, daß die dritten Schnittpunkte der Seiten von $B_{1}, B_{2}, B_{3}$ die Eckpunkte von $A^{\prime}$ bilden; dieselben Eckpunkte von $A^{\prime}$ sind zugleich aber auch die Tangentialpunkte der Eckpunkte von $B_{1}, B_{2}, B_{3}$ : ist also $a_{p}^{\prime}$ ein solcher Eckpunkt von $A^{\prime}$, so ist eine der vier von $a_{p}^{\prime}$ ausgehenden weiteren Tangenten die Tangente $a_{p-1}^{\prime} a_{p}^{\prime}$, deren Berührungspunkt $a_{p-1}^{\prime}$ wieder ein Eckpunkt von $A^{\prime}$ ist; von den drei anderen Berührungspunkten $b_{p-1}^{\prime}, b_{p-1}^{\prime \prime}, b_{p-1}^{\prime \prime \prime}$ gehört jeder einem der drei Vielecke $B_{1}, B_{2}, B_{3}$ als Eckpunkt an:

Die drei Vielecke

$$
\begin{aligned}
& B_{1}\left(b_{1}^{\prime} b_{2}^{\prime} \ldots b_{p-1}^{\prime} b_{p}^{\prime} b_{p+1}^{\prime} \ldots\right) \\
& B_{2}\left(b_{1}^{\prime \prime} b_{2}^{\prime \prime} \ldots b_{p-1}^{\prime \prime} b_{p}^{\prime \prime} b_{p+1}^{\prime \prime} \cdots\right) \\
& B_{3}\left(b_{1}^{\prime \prime \prime} b_{2}^{\prime \prime \prime} \ldots b_{p+1}^{\prime \prime \prime} b_{p}^{\prime \prime \prime} b_{p+1}^{\prime \prime \prime} \cdots\right)
\end{aligned}
$$

stehen in der Beziehung zueinander, daß z. B. die Geraden $b_{p-1}^{\prime} b_{p-1}^{\prime \prime}, b_{p+1}^{\prime} b_{p}^{\prime \prime}$ und $b_{p}^{\prime} b_{p+1}^{\prime \prime}$ alle drei durch $b_{p}^{\prime \prime \prime}$ gehen. Drücken wir das letztere wieder in der Sprache unserer Analogie aus, so können wir kurz sagen: Die polygonalen Punkte $B_{1}, B_{2}, B_{3}$ ergeben die harmonische Polare zu dem polygonalen Wendepunkt $A$. 